\title{
III. The impulsive motion of an electrified sphere
}

\section{G.F.C. Searle M.A. F.R.S.}

To cite this article: G.F.C. Searle M.A. F.R.S. (1909) III. The impulsive motion of an electrified sphere, Philosophical Magazine Series 6, 17:97, 43-71, DOI: $10.1080 / 14786440108636580$

To link to this article: http://dx.doi.org/10.1080/14786440108636580

$$
\text { 册 Published online: } 21 \text { Apr } 2009 .
$$

Submit your article to this journal $\pi$

LII Article views: 3

Q View related articles $\asymp$ 
With $C_{1}=11.87 \mathrm{mfd}$. the frequency of the arc note was 578 , and of the damped oscillation 678 . In these cases also the arc frequencies are considerably less than those of the same circuits without the arc.

With $\mathrm{C}_{1}=9 \cdot 55$ and $\mathrm{C}_{2}$ about $\cdot 000275 \mathrm{mfd}$. one note was heard, and the secondary potential reached very high values during the oscillations, as was shown by copious "brushing" at the plates of the secondary condenser. The curve is shown in Pl. I. fig. 11. The amplitude was at times even greater than is there shown, and the zero-line had to be brought below the tuning-fork wave in order to keep the vibrating ray on the rotating mirror. The frequency is 613 , and the octave appears to have an amplitude several times as great as that of the fundamental. The value of $\mathrm{L}_{1} \mathrm{C}_{1}$ is $4 \cdot 411 \times 10^{-8}$, of $\mathrm{L}_{2} \mathrm{C}_{2}$ about $1.929 \times 10^{-8}$; the frequencies calculated by (4) from the inductances and capacities are approximately 723 and 1300 . The high potential at the plates of the secondary condenser in this case appears to be due to approximate agreement in frequency between the octave of the arc note and the higher natural vibration of the secondary coil. Similar evidences of "resonance" of a transitory kind were frequently observed with the other condensers when the arc note (either the high note or the low note) during its fluctuations fell rather low in pitch. All the effects here described may also be conveniently obtained by using a variable condenser with oil dielectric as the secondary capacity.

Bangor, September 1908.

III. The Impulsive Motion of an Electrified Sphere. By G. F. C. SeARLe, M.A., F.R.S., University Lecturer in Experimental P'hysics, Cambridge*.

$\S 1$. $\mathrm{T}$ the end of a paper on "The Impulsive Motion of A Electrified Systems," + published in the Philosophical Magazine for January 1907, I have calculated the energy and momentum radiated when the velocity of a

* Commonicated by the Author.

+ I regret that when I wrote this paper I was unaware that Dr. Paul Hertz had obtained some of the results in his Untersuchumgen über unstetige Bewegungen eines Electrons, Göttingen, 1904. His paper was in my possessiun at the time, but $I$ was under the impression that it referred to the force required to set a charged sphere in motion and not to the problems which I was considering. Where my work overlaps that of Dr. Hertz, it gives an independent confirmation of his results, as it was done without any reference to his paper. 
charged sphere is impulsively changed by an infinitesimal amount, the change of velocity making any angle with the initial velocity. In the present paper I complete the investigation by calculating the energy and momentum which are radiatod when the velocity of the sphere is impulsively changed in any manner, the only restrictions being that both the initial and the final velocities are less than that of light, and that the sphere has no angular velocity about any axis through its centre. The results in the restricted case, in which the centre of the sphere moves along a single straight line throughout, have been given by Heaviside and by Paul Hertz.

$\S 2$. The investigation turns mainly upon a certain integral. In the evaluation of this integral I have been greatly aided by Mr. G. T. Bennett, Fellow of Emmanuel College. I had carried out the integration in the manner given in $\$ 10$ below, but I was unable to put the result into a symmetrical form. Mr. Bennett then devised the very elegant semi-geometrical process which is given in $\$ \S 6,7,8$, and, guided by the result, reduced the complicated expression arising in my method of integration to a symmetrical form. I am also indebted to Mr. F.J. W. Whipple, of the Merchant Taylors' School, for verifying the integration by an alternative method.

$\S 3$. Now let us consider a sphere of radius $a$, carrying a charge $Q$ on its surface, and let us suppose that its velocity is impulsively changed from $\mathbf{u}_{1}$ to $\mathbf{u}_{2}$, and that the angle between $\mathfrak{u}_{1}$ and $\mathrm{u}_{2}$ is $\alpha$, heary type denoting vectors. Then it will be seen from $\S 31$ of my earlier paper that, when the pulse generated by the change of velocity has travelled out through a distance $r$, which is very great compared with the diameter of the sphere, the electric force in the pulse is at right angles to the radius and is given by

$$
\mathbf{E}=\frac{\mathrm{Q}}{2 \mathrm{~K} r a}\left\{\frac{\mathbf{R}\left(\mathbf{u}_{2} \mathbf{R}\right)-\mathbf{u}_{2}}{v-\mathfrak{u}_{2} \mathbf{R}}-\frac{\mathbf{R}\left(\mathfrak{u}_{1} \mathbf{R}\right)-\mathfrak{u}_{1}}{v-\mathbf{u}_{1} \mathbf{R}}\right\} \ldots .
$$

Here $\mathbf{R}$ denotes, for the moment, a unit vector along the radius, and $\mathbf{u}_{1} \mathbf{R}$ denotes the scalar product of $\mathbf{u}_{1}$ and $\mathbf{R}$, while $K$ is the specific inductive capacity and $v$ the velocity of light.

The magnetic force in the pulse is at right angles to both $\mathbf{E}$ and $\mathbf{R}$, and is given by

$$
\mathbf{H}=v \mathrm{KVRE}, \quad \cdots \quad \cdot \quad \cdot \quad \cdot
$$

where VRE is a vector product. 
If $\theta_{1}$ and $\theta_{2}$ be the angles between the radius and the directions of $\mathbf{u}_{1}$ and $\mathbf{u}_{2}$, we have $\mathbf{u}_{1} \mathbf{R}=u_{1} \cos \theta_{1}$ and $\mathbf{u}_{2} \mathbf{R}=u_{2} \cos \theta_{2}$, and $\mathfrak{u}_{1} \mathfrak{u}_{2}=u_{1} t_{2} \cos \alpha$, where $\alpha$ is the angle between $\mathfrak{u}_{1}$ and $\mathfrak{u}_{2}$. Thus we find

$$
\begin{aligned}
\mathrm{E}^{2}=\frac{\mathrm{Q}^{2}}{4 \mathrm{~K}^{2} r^{2} a^{2}}\left\{\frac{u_{1}^{2} \sin ^{2} \theta_{1}}{\left(v-u_{1} \cos \theta_{1}\right)^{2}}+\frac{u_{2}^{2} \sin ^{2} \theta_{2}}{\left(v-u_{2} \cos \ddot{\theta}_{2}\right)^{2}}\right. \\
\left.-2 \frac{u_{1} u_{2} \cos \alpha-u_{1} u_{2} \cos \theta_{1} \cos \theta_{2}}{\left(v-u_{1} \cos \theta_{1}\right)\left(v-z_{2} \cos \theta_{2}\right)}\right\} .
\end{aligned}
$$

The numerator of the last term in this expression can be written in the form

$$
\begin{array}{r}
2\left\{v\left(v-u_{1} \cos \theta_{1}\right)+v\left(v-u_{2} \cos \theta_{2}\right)-\left(v-u_{1} \cos \theta_{1}\right)\left(v-u_{2} \cos \theta_{2}\right)\right. \\
\left.-v^{2}+u_{1} u_{2} \cos \alpha\right\},
\end{array}
$$

and thus we obtain, after an easy reduction,

$$
\begin{array}{r}
\mathrm{E}^{2}=\frac{\mathrm{Q}^{2}}{4 \mathrm{~K}^{2} r^{2} a^{2}}\left\{\frac{2\left(v^{2}-u_{1} u_{2} \cos \alpha\right)}{\left(v-u_{1} \cos \theta_{1}\right)\left(v-u_{2} \cos \theta_{2}\right)}-\frac{v^{2}-u_{1}{ }^{2}}{\left(v-u_{1} \cos \theta_{1}\right)^{2}}\right. \\
\left.-\frac{v^{2}-u_{2}{ }^{2}}{\left(v-u_{2} \cos \theta_{2}\right)^{2}}\right\} \text {. . . . }
\end{array}
$$

In the pulse, the magnetic energy per unit volume is equal to the electric energy per unit volume, and hence, remembering that the thickness of the pulse is $2 a$, we find that $\mathrm{W}$, the radiated energy, is given by

$$
\mathrm{W}=\frac{\mathrm{K} a r^{2}}{2 \pi} \int \mathrm{E}^{2} d \omega, \quad . \quad . \quad . \quad .
$$

where $d \omega$ is an element of solid angle.

The last two terms in the expression for $\mathrm{E}^{2}$ can be integrated at once. Thus we have

$$
\begin{aligned}
\int \frac{d \omega}{\left(v-u_{1} \cos \theta_{1}\right)^{2}} & =2 \pi \int_{0}^{\pi} \frac{\sin \theta_{1} d \theta_{1}}{\left(v-u_{1} \cos \theta_{1}\right)^{2}} \\
& =\frac{4 \pi}{v^{2}-u_{1}^{2}} .
\end{aligned}
$$

The expression for W now becomes

$$
W=\frac{Q^{2}}{\delta \pi a \mathrm{~K}}\left\{\int \frac{2\left(v^{2}-u_{1} u_{2} \cos \alpha\right) d \omega}{\left(v-u_{1} \cos \theta_{1}\right)\left(v-u_{2} \cos \theta_{2}\right)}-\delta \pi\right\} .
$$

Writing $n_{1}$ for $u_{1} / v$ and $n_{2}$ for $u_{2} / v$, and noting that 
$\mathrm{Q}^{2} / 2 a \mathrm{~K}=\mathrm{U}_{0}$, the electric energy of the sphere at rest, we have

$$
\mathrm{W}=\mathrm{U}_{0}\left\{\frac{1-n_{1} n_{2} \cos \alpha}{2 \pi} \int \frac{d \omega}{\left(1-n_{1} \cos \theta_{1}\right)\left(1-n_{2} \cos \theta_{2}\right)}-2\right\}
$$

The only difficulty lies in the evaluation of the integral in this expression. Since the integral is a function of $n_{1}, n_{2}$, and $\alpha$, we shall write

$$
\mathrm{F}\left(n_{1}, n_{2}, \alpha\right)=\int \frac{d \omega}{\left(1-n_{1} \cos \theta_{1}\right)\left(1-n_{2} \cos \theta_{2}\right)},
$$

where the integration extends over the surface of the unit sphere of which $d \omega$ is an element. Thus

$$
\mathrm{W}=\mathrm{U}_{0}\left\{\left(1-n_{1} n_{2} \cos \alpha\right)(2 \pi)^{-1} \mathrm{~F}\left(n_{1}, n_{2}, \alpha\right)-2\right\} .
$$

$\S 4$. When the directions of $\mathfrak{u}_{1}$ and $\mathfrak{u}_{2}$ are parallel and in the same sense, so that $\cos \alpha=1$, the value of $F$ is easily found, for we now have $\theta_{2}=\theta_{1}$ and can take $2 \pi \sin \theta_{1} d \theta_{1}$ as the element of surface of the unit sphere. Thus

$$
\begin{aligned}
F\left(n_{1} n_{2}, 0\right) & =2 \pi \int_{0}^{\pi} \frac{\sin \theta_{1} d \theta_{1}}{\left(1-n_{1} \cos \theta_{1}\right)\left(1-n_{2} \cos \theta_{1}\right)} \\
& =\frac{2 \pi}{n_{1}-n_{2}}\left\{n_{1} \int_{0}^{\pi} \frac{\sin \theta_{1} d \theta_{1}}{1-n_{1} \cos \theta_{1}}-n_{2} \int_{0}^{\pi} \frac{\sin \theta_{1} d \theta_{1}}{1-n_{2} \cos \theta_{1}}\right\} \\
& =\frac{2 \pi}{n_{1}-n_{2}} \log \frac{\left(1+n_{1}\right)\left(1-n_{2}\right)}{\left(1-n_{1}\right)\left(1+n_{2}\right)} . \quad . \quad . \quad . \quad . \quad(9)
\end{aligned}
$$

When $u_{1}$ and $u_{2}$ are parallel but directed in opposite directions, so that $\cos \alpha=-1$, we have $\cos \theta_{2}=-\cos \theta_{1}$, and hence the value of $\mathrm{F}$ can be found from (9) by changing the sign of $n_{2}$. Then

$$
\mathrm{F}\left(n_{1}, n_{2}, \pi\right)=\frac{2 \pi}{n_{1}+n_{2}} \log \frac{\left(1+n_{1}\right)\left(1+n_{2}\right)}{\left(1-n_{1}\right)\left(1-n_{2}\right)} .
$$

Substituting these values of $\mathrm{F}$ in (8) we obtain the following expressions for the radiated energy :-

When $\mathfrak{u}_{1}$ and $\mathfrak{u}_{2}$ are parallel and in the same direction, so that $\cos \alpha=1$,

$$
\mathrm{W}=\mathrm{U}_{0}\left\{\frac{1-n_{1} n_{2}}{n_{1}-n_{2}} \log \frac{\left(1+n_{1}\right)\left(1-n_{2}\right)}{\left(1-n_{1}\right)\left(1+n_{2}\right)}-2\right\} .
$$


When $\mathfrak{u}_{1}$ and $\mathfrak{u}_{2}$ are parallel but in opposite directions, so that $\cos a=-1$,

$$
\mathrm{W}=\mathrm{U}_{0}\left\{\frac{1+n_{1} n_{2}}{n_{1}+n_{2}} \log \frac{\left(1+n_{1}\right)\left(1+n_{2}\right)}{\left(1-n_{1}\right)\left(1-n_{2}\right)}-2\right\} . .
$$

When the motion is exactly reversed, so that $\cos x=-1$ and $n_{2}=n_{1}$

$$
\mathrm{W}=\mathrm{U}_{0}\left\{\frac{1+n_{1}^{2}}{n_{1}} \log \frac{1+n_{1}}{1-n_{1}}-2\right\} . \quad . \quad . \quad .
$$

The first result (11) was given by Dr. Oliver Heaviside*. These results have also been deduced by Paul Hertz $\dagger$ on dynamical principles from the values of the energy and momentum of a charged sphere in steady rectilinear motion.

$\S 5$. We now pass on to the general case in which the initial and final velocities are inclined at any angle. The integration of $\mathrm{F}\left(n_{1}, n_{2}, \alpha\right)$ is now somewhat complicated, but on account of the fundamental importance of the integral two independent methods of evaluation are given below.

\$ 6 . The first method of evaluating $\mathrm{F}\left(n_{1}, n_{2}, \alpha\right)$ is due to Mr.G. T. Bennett. Since this method is partly geometrical, it will be convenient to modify the integral so as to exhibit the geometrical quantities involved. We see, at once, from (7) that

$$
\mathrm{F}\left(\frac{\mathrm{R}}{h_{1}}, \frac{\mathrm{R}}{h_{2}}, \alpha\right)=\frac{h_{1} h_{2}}{\mathrm{R}^{2}} \cdot \mathrm{J}, \ldots . \cdot
$$

where

$$
\mathrm{J}=\int^{0} \frac{\mathrm{R}^{2} d \omega}{\left(h_{1}-\mathrm{R} \cos \theta_{1}\right)\left(h_{2}-\mathrm{R} \cos \theta_{2}\right)} \cdot \cdots
$$

Here $\theta_{1}$ and $\theta_{2}$ are the angles between the radius defined by $d \omega$ and two fixed radii drawn from the centre of a sphere of radius $R$, and thus we can write

$$
\mathrm{J}=\int \frac{\mathrm{R}^{2} d \omega}{z_{1} z_{2}}, \quad \cdot \quad \cdot \quad \cdot \quad \cdot \quad \cdot
$$

where $z_{1}, z_{2}$ are the perpendiculars drawn from any point on the surface of the sphere upon two fixed planes which cut the two fixed radii at right angles at distances $h_{1}$ and $h_{2}$ from the centre of the sphere. Since $n_{1}<1$ and $n_{2}<1$ we suppose

* 'Nature,' Nov. 6, 1902.

+ Untersuchungen über unstetige Bewegnngen eines Electrons. Göttingen, 1904. See also M. Abraham, Theorie der Electrizität, vol. ii. p. 233 . 
that $h_{1}>\mathrm{R}$ and $h_{2}>\mathrm{R}$, and thus the planes do not cut the sphere.

If we denote the mean value of $1 / \tilde{z}_{1} \tilde{z}_{2}$ for the surface of the sphere by $\left[1 / z_{1} z_{2}\right]_{\mathrm{s}}$, we have

$$
\mathrm{J}=4 \pi \mathrm{R}^{2}\left[\frac{1}{z_{1} z_{2}}\right]_{\mathrm{s}} \cdot \quad \cdot \quad \cdot \quad \cdot \quad \cdot
$$

The first step is to eut the sphere in a circle by a plane perpendicular to both the fixed planes and to find the mean value of $1 / z_{1} z_{2}$ for this circle. The case in which the two fixed planes are parallel has been discussed in $\S 4$. We may therefore now suppose that their line of intersection is at a finite distance from the centre of the sphere.

$\S 7$. Let the plane of the paper be normal to the line of intersection of the two fixed planes and let it cut the sphere in a circle of radius $r$, the centre of the circle being at a distance $\mathrm{L}$ from the line of intersection. Let $\mathrm{OH}_{1}, \mathrm{OH}_{2}$ (fig. 1) be sections of the two fixed planes by the plane of

Fig: 1.

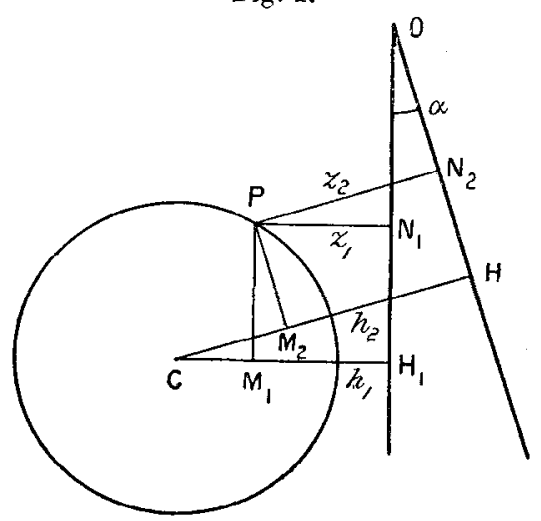

the paper, and let $\mathrm{CH}_{1}=h_{1}$ and $\mathrm{CH}_{2}=h_{2}$ be the perpendiculars from $\mathrm{C}$, the centre of the circle, upon $\mathrm{OH}_{1}$ and $\mathrm{OH}_{2}$. Then the angles $\mathrm{H}_{1} \mathrm{CH}_{2}$ and $\mathrm{H}_{1} \mathrm{OH}_{2}$ are each equal to $\alpha$. If $\mathrm{P}$ be any point on the circle and $\mathrm{PN}_{1}, \mathrm{PN}_{2}$ be perpendiculars from $\mathrm{P}$ upon the lines $\mathrm{OH}_{1}, \mathrm{OH}_{2}$, then $\mathrm{PN}_{1}=z_{1}$ and $\mathrm{PN}_{2}=z_{2}$.

Now draw $\mathrm{PM}_{1}$ and $\mathrm{PM}_{2}$ perpendicular to $\mathrm{CH}_{1}$ and $\mathrm{CH}_{2}$. Then $\mathrm{CM}_{1}=h_{1}-z_{1}$ and $\mathrm{CM}_{2}=h_{2}-z_{2}$. Since $\mathrm{M}_{1}$ and $\mathrm{M}_{2}$ lie on a circle of which $\mathrm{CP}$ is the diameter, and since $\mathbf{M}_{1} \mathbf{M}_{2}$ subtends the angle $\alpha$ at $C$, it follows that $M_{1} M_{2}$ subtends an angle $2 \alpha$ at the middle point of CP. Hence

$$
\mathrm{M}_{1} \mathrm{M}_{2}=\mathrm{CP} \sin \alpha=r \sin \alpha \text {. }
$$


Since $\mathrm{CO}=\mathrm{L}$, we find in a similar manner

$$
\mathrm{H}_{1} \mathrm{H}_{2}=\mathrm{L} \sin \alpha \text {. }
$$

From the triangles $\mathrm{M}_{1} \mathrm{CM}_{2}$ and $\mathrm{H}_{1} \mathrm{CH}_{2}$ we have

$$
\begin{aligned}
r^{2} \sin ^{2} \alpha & =\left(h_{1}-z_{1}\right)^{2}+\left(h_{2}-z_{2}\right)^{2}-2\left(h_{1}-z_{1}\right)\left(h_{2}-z_{2}\right) \cos \alpha, \\
\mathrm{L}^{2} \sin ^{2} \alpha & =h_{1}^{2}+h_{2}^{2}-2 h_{1} h_{2} \cos \alpha ;
\end{aligned}
$$

and hence

$$
\begin{aligned}
& \left(\mathrm{L}^{2}-r^{2}\right) \sin ^{2} \alpha=2 z_{1} z_{2} \cos \alpha+2 z_{1}\left(h_{1}-h_{2} \cos \alpha\right) \\
& \text { Dividing by } z_{1} z_{2} \text {, we obtain } \quad+2 z_{2}\left(h_{2}-h_{1} \cos \alpha\right)-z_{1}{ }^{2}-z_{2}{ }^{2} . \\
& \frac{\left(\mathrm{L}^{2}-r^{2}\right) \sin ^{2} \alpha}{z_{1} z_{2}{ }^{2}}=2 \cos \alpha+\frac{2\left(h_{1}-h_{2} \cos \alpha\right)}{z_{2}}+\frac{2\left(h_{2}-h_{1} \cos \alpha\right)}{z_{1}} \\
& -\frac{z_{1}}{z_{2}}-\frac{z_{2}}{z_{1}} \cdot \quad \text { (18) }
\end{aligned}
$$

The problem of finding the mean value of $1 / \tilde{z}_{1} \tilde{z}_{2}$ for the circle is thus reduced to finding the mean values of $1 / z_{1}$, $1 / z_{2}, z_{1} / z_{2}$ and $z_{2} / z_{1}$ for the same circle.

If $\left[1 / z_{1}\right]_{c}$ denote the mean value of $1 / z_{1}$ for the circle of radius, $r$, and if $\phi_{1}$ denote the angle $\mathrm{PCH}_{1}$, we have

$$
\left[\frac{1}{z_{1}}\right]_{\mathrm{C}}=\frac{1}{\pi r} \int_{0}^{\pi} \frac{r d \phi_{1}}{h_{1}-r \cos \phi_{1}},
$$

since the integral from $\pi$ to $2 \pi$ is equal to that from 0 to $\pi$. Putting $\tan \frac{1}{2} \phi_{1}=x$ and therefore $\cos \phi_{1}=\left(1-x^{2}\right) /\left(1+x^{2}\right)$, we find

$$
\begin{aligned}
{\left[\frac{1}{z_{1}}\right]_{\mathrm{C}} } & =\int_{0}^{\infty} \frac{2 d x / \pi}{h_{1}-r+\left(h_{1}+r\right) x^{2}}=\frac{2 / \pi}{\left(h_{1}^{2}-r^{2}\right)^{\frac{1}{2}}}\left[\tan ^{-1} x \sqrt{\frac{h_{1}+r}{h_{1}-r}}\right]_{0}^{\infty} \\
& =\frac{1}{\left(h_{1}^{2}-r^{2}\right)^{\frac{1}{2}}}=\frac{1}{t_{1}}, . . . . . . . . . . .
\end{aligned}
$$

where $t_{1}$ is the length of the tangent from $\mathrm{H}_{1}$ to the circle,

Similarly

$$
\left[1 / z_{2}\right]_{\mathrm{C}}=1 / t_{2} \text {. . . . . . }
$$

Now

$$
\frac{z_{2}}{z_{1}}=\frac{\mathrm{PN}_{2}}{\mathrm{PN}_{1}}=\frac{\mathrm{PN}_{1} \cos \alpha+\mathrm{ON}_{1} \sin \alpha}{\mathrm{PN}_{1}} \text {. }
$$

But there are two positions of $\mathrm{P}$ for which $\mathrm{PN}_{1}$ or $z_{1}$ has the same value, and the average value of $\mathrm{ON}_{1}$ for these two positions is $\mathrm{OH}_{1}$. Thus we find for the whole circle

$$
\begin{aligned}
{\left[\begin{array}{c}
z_{2} \\
z_{1}
\end{array}\right]_{\mathrm{C}} } & =\cos \alpha+\mathrm{OH}_{1} \sin \alpha\left[\frac{1}{z_{1}}\right]_{\mathrm{C}} \\
& =\cos \alpha+\sin \alpha \cdot \mathrm{OH}_{1} / t_{1} .
\end{aligned}
$$

Phil. Mag. S. 6. Vol. 17. No. 97. Jan. 1909. 
Similarly, since

we find

$$
\frac{z_{1}}{z_{2}}=\frac{\mathrm{PN}_{1}}{\mathrm{PN}_{2}}=\frac{\mathrm{PN}_{2} \cos \alpha-\mathrm{ON}_{2} \sin \alpha}{\mathrm{PN}_{2}},
$$

$$
\left[\frac{z_{1}}{z_{2}}\right]_{\mathrm{C}}=\cos \alpha-\sin \alpha \cdot \mathrm{OH}_{2} / t_{2} .
$$

But $\quad \mathrm{OH}_{1} \sin \alpha=\mathrm{CH}_{2}-\mathrm{CH}_{1} \cos \alpha=h_{2}-h_{1} \cos \alpha$ and thus

$$
\mathrm{OH}_{2} \sin \alpha=\mathrm{CH}_{2} \cos \alpha-\mathrm{CH}_{1}=h_{2} \cos \alpha-h_{1} \text {, }
$$

$$
\begin{aligned}
& {\left[z_{2} / z_{1}\right]_{\mathrm{C}}=\cos \alpha+\left(h_{2}-h_{1} \cos \alpha\right) / t_{1} \quad . .} \\
& {\left[z_{1} / z_{2}\right]_{\mathrm{C}}=\cos \alpha+\left(h_{1}-h_{2} \cos \alpha\right) / t_{2} .}
\end{aligned}
$$

When we substitute in (18) the mean values shown in (19), $(20),(21)$, and (22), we obtain

$$
\left(\mathrm{L}^{2}-r^{2}\right) \sin ^{2} \alpha\left[\frac{1}{z_{1} z_{2}}\right]_{\mathrm{C}}=\frac{h_{1}-h_{2} \cos \alpha}{t_{2}}+\frac{h_{2}-h_{1} \cos \alpha}{t_{1}} .
$$

But $h_{2}-h_{1} \cos \alpha=\mathrm{OH}_{1} \sin \alpha=\sin \alpha \cdot\left(\mathrm{L}^{2}-h_{1}^{2}\right)^{\frac{1}{2}}$. .

$$
h_{2} \cos \alpha-h_{1}=\mathrm{OH}_{2} \sin \alpha=\sin \alpha\left(\mathrm{L}^{2}-h_{2}^{2}\right)^{\frac{1}{2}}, \text {. . }
$$

and hence

$$
\left[\frac{1}{z_{1} z_{2}}\right]_{\mathrm{C}}=\frac{1}{\left(\mathrm{~L}^{2}-r^{2}\right) \sin \alpha}\left\{\frac{1}{t_{1}}\left(\mathrm{~L}^{2}-h_{1}^{2}\right)^{\frac{2}{2}}-\frac{1}{t_{2}}\left(\mathrm{~L}^{2}-h_{2}^{2}\right)^{\frac{1}{2}}\right\} \text {. }
$$

$\S 8$. We can now find the mean value of $1 / z_{1} z_{2}$ for the surface of the sphere. If we measure $x$ from the centre of the sphere parallel to the line of intersection of the two fixed planes, the area of the zone on the sphere defined by $x$ and $x+d x$ is $2 \pi \mathrm{R} d x$, and thus

$$
\left[\frac{1}{z_{1} z_{2}}\right]_{\mathrm{s}}=\frac{1}{2 \pi \mathrm{R}^{2}} \int_{0}^{\mathrm{R}}\left[\frac{1}{z_{1} z_{2}}\right]_{\mathrm{C}} 2 \pi \mathrm{R} d x .
$$

Now

$$
r^{2}=\mathrm{R}^{2}-x^{2}
$$

and hence

$$
\begin{aligned}
& t_{1}^{2}=h_{1}^{2}-r^{2}=h_{1}^{2}-\mathrm{R}^{2}+x^{2}, \\
& t_{2}^{2}=h_{2}^{2}-r^{2}=h_{2}^{2}-\mathrm{R}^{2}+x^{2} .
\end{aligned}
$$

Thus we have

$$
\begin{array}{r}
{\left[\frac{1}{z_{1} z_{2}}\right]_{\mathrm{s}}=\frac{1}{\mathrm{R} \sin \alpha} \int_{0}^{\mathrm{R}} \frac{d x}{\mathrm{~L}^{2}-\mathrm{R}^{2}+x^{2}}\left\{\frac{\left(\mathrm{L}^{2}-h_{1}^{2}\right)^{\frac{1}{2}}}{\left(h_{1}^{2}-\mathrm{R}^{2}+x^{2}\right)^{\frac{1}{2}}}\right.} \\
\left.-\frac{\left(\mathrm{L}^{2}-h_{2}^{2}\right)^{\frac{1}{2}}}{\left(h_{2}^{2}-\mathrm{R}^{2}+x^{2}\right)^{\frac{1}{2}}}\right\} .
\end{array}
$$


The integration can be effected by aid of the formula

$$
\int \frac{\left(p^{2}-q^{2}\right)^{\frac{1}{2}} d x}{\left(p^{2}+x^{2}\right)\left(q^{2}+x^{2}\right)^{\frac{1}{2}}}=\frac{1}{2 p} \log \frac{p\left(q^{2}+x^{2}\right)^{\frac{1}{2}}+x\left(p^{2}-q^{2}\right)^{\frac{1}{2}}}{p\left(q^{2}+x^{2}\right)^{\frac{1}{2}}-x\left(p^{2}-q^{2}\right)^{\frac{1}{2}}}
$$

if wo put $p^{2}=\mathrm{L}^{2}-\mathbf{R}^{2}, q^{2}=h^{2}-\mathbf{R}^{2}$,

and $\quad p^{2}-q^{2}=\mathrm{L}^{2}-h^{2}$,

where $h$ stands for either $h_{1}$ or $h_{2}$. We thus obtain

where

$$
\left[\frac{1}{z_{1} z_{2}}\right]_{\mathrm{s}}=\frac{1}{2 \mathrm{R} \sin \alpha\left(\mathrm{L}^{2}-\mathrm{R}^{2}\right)^{\frac{1}{2}}}[\log \mathrm{A}-\log \mathrm{B}]_{0}^{\mathrm{R}},
$$

Thus

$$
\dot{\mathrm{A}}=\frac{\left(\mathrm{L}^{2}-\mathrm{R}^{2}\right)^{\frac{1}{2}}\left(h_{1}^{2}-\mathrm{R}^{2}+x^{2}\right)^{\frac{1}{2}}+x\left(\mathrm{~L}^{2}-h_{1}^{2}\right)^{\frac{1}{2}}}{\left(\mathrm{~L}^{2}-\mathrm{R}^{2}\right)^{\frac{1}{2}}\left(h_{1}^{2}-\mathrm{R}^{2}+x^{2}\right)^{\frac{1}{2}}-x\left(\mathrm{~L}^{2}-h_{1}^{2}\right)^{\frac{1}{2}}}
$$

$$
[\log \mathrm{A}]_{0}^{\mathrm{R}}=\log \frac{h_{1}\left(\mathrm{~L}^{2}-\mathrm{R}^{2}\right)^{\frac{1}{2}}+\mathrm{R}\left(\mathrm{L}^{2}-h_{1}{ }^{2}\right)^{\frac{1}{2}}}{h_{1}\left(\mathrm{~L}^{2}-\mathrm{R}^{2}\right)^{\frac{1}{2}}-\mathrm{R}\left(\mathrm{L}^{2}-h_{1}\right)^{\frac{1}{2}}}
$$

For $\mathrm{B}$ we have only to substitute $h_{2}$ for $h_{1}$. Hence

$$
\begin{aligned}
& {\left[\log \frac{\mathrm{A}}{\mathrm{B}}\right]_{0}^{\mathrm{R}}=} \\
& \quad \log \frac{\left.\left\{h_{1}\left(\mathrm{~L}^{2}-\mathrm{R}^{2}\right)^{\frac{1}{3}}+\mathrm{R}^{\prime} \mathrm{L}^{2}-h_{1}^{2}\right)^{\frac{1}{2}}\right\}\left\{h_{2}\left(\mathrm{~L}^{2}-\mathrm{R}^{2}\right)^{\frac{1}{2}}-\mathrm{R}\left(\mathrm{L}^{2}-h_{2}^{2}\right)^{\frac{1}{2}}\right\}}{\left\{h_{1}\left(\overline{\mathrm{L}^{2}}-\mathrm{R}^{2}\right)^{\frac{1}{2}}-\mathrm{R}\left(\mathrm{L}^{2}-h_{1}^{2}\right)^{\frac{1}{2}}\right\}\left\{h_{2}\left(\mathrm{~L}^{2}-\mathrm{R}^{2}\right)^{\frac{1}{2}}+\mathrm{R}\left(\mathrm{L}^{2}-h_{2}^{2}\right)^{\frac{1}{2}}\right\}} .
\end{aligned}
$$

We now substitute for $\left(\mathrm{L}^{2}-h_{1}{ }^{2}\right)^{\frac{1}{2}}$ and $\left(\mathrm{L}^{2}-h_{2}^{2}\right)^{\frac{1}{2}}$ the values given in (23) and (24), and then carry out the multiplications in the numerator and denominator of the quaniity under the logarithm. Replacing $h_{1}{ }^{2}+h_{2}{ }^{2}-2 h_{1} h_{2} \cos \alpha$ by $\mathrm{L}^{2} \sin ^{2} \alpha$, we then easily find

$$
\begin{aligned}
& {\left[\frac{1}{z_{1} z_{2}}\right]_{\mathrm{s}}=\frac{1}{2 \overline{\mathrm{R}} \sin \alpha\left(\mathrm{L}^{2}-\mathrm{R}^{2}\right)^{\frac{1}{2}}}\left[\log \frac{\mathrm{A}}{\mathrm{B}}\right]_{0}^{\mathbf{R}}} \\
& =\frac{1}{2 \mathrm{R}} \frac{1}{\sin \alpha\left(\mathrm{L}^{2}-\mathrm{R}^{2}\right)^{\frac{1}{2}}} \log \frac{h_{1}}{\frac{h_{2}}{h_{1}} h \mathrm{R}_{2}-\cos \alpha+\mathrm{R}\left(\mathrm{L}^{2}-\mathrm{R}^{2}\right)^{\frac{1}{2}} \sin \alpha}
\end{aligned}
$$

where $\mathrm{L}^{2} \sin ^{2} \alpha=h_{1}^{2}+h_{2}{ }^{2}-2 h_{1} h_{2} \cos \alpha$.

We have thus found the mean value of $1 / z_{1} z_{2}$ for points on the surface of the sphere.

Mr. Bennett has supplied the following remarks :- "The quantity under the logarithm in (26) is the cross-ratio of the pencil formed by the two planes together with the two tangent planes to the sphere through their line of intersection; and the result takes a simpler form if reciprocated with respect to the sphere itself. The perpendiculars from a variable point of the sphere to fixed planes are then replaced by (constant multiples of) perpendiculars drawn from fixed points within the sphere to a variable tangent plane. The result of the E 2 
mean-value problem may then be expressed concisely as follows :-

A sphere being given, $\mathrm{AB}$ being a fixed chord, and $\mathrm{CD}$ being fixed points on the chord, the mean value of the reciprocal of the product of the distances of $\mathrm{C}$ and $\mathrm{D}$ from a variable tangent plane is $\log (\mathrm{ABCD}) \div \mathrm{AB}$. CD."

\$ 9. To apply (26) to the electrical problem, wo put

$$
\mathrm{R}=1, \quad h_{1}=1 / n_{1}=v / u_{1}, \quad h_{2}=1 / n_{2}=v / u_{2},
$$

It will be convenient to write

$$
n_{1}^{2}+n_{2}^{2}-2 n_{1} n_{2} \cos \alpha=m^{2}=w^{2} / v^{2}, \quad .
$$

where $w$ is the change of the velocity of the sphere, so that $\mathfrak{u}_{2}$ is the resultant of $\mathfrak{u}_{1}$ and $\mathbf{w}$. Then

and

$$
\mathrm{L}^{2} \sin ^{2} \alpha=\frac{1}{n_{1}^{2}}+\frac{1}{n_{2}^{2}}-\frac{2 \cos \alpha}{n_{1} n_{2}}=\frac{m^{2}}{n_{1}^{2} n_{2}^{2}}
$$

By $\& 6$ wo have

$$
\left(\mathrm{L}^{2}-\mathrm{R}^{2}\right) \sin ^{2} \alpha=\frac{m^{2}-n_{1}^{2} n_{2}^{2} \sin ^{2} \alpha}{n_{1}^{2} n_{2}^{2}} .
$$

$$
\mathrm{F}\left(n_{1}, n_{2}, a\right)=\frac{4 \pi}{n_{1} n_{2}}\left[\frac{1}{z_{1} z_{2}}\right]_{\mathrm{s}}
$$

provided $h_{1}, h_{2}$ and $\mathrm{R}$ have the values just written. Inserting those values in (26) we have

$\mathrm{F}\left(n_{1}, n_{2}, a\right)=$

$$
\frac{2 \pi}{\left(m^{\overline{2}}-n_{1}^{2} n_{2}^{2} \sin ^{2} \alpha\right)^{\frac{1}{2}}} \log \frac{1-n_{1} n_{2} \cos \alpha+\left(m^{2}-n_{1}^{2} n_{2}{ }^{2} \sin ^{2} \alpha\right)^{\frac{2}{2}}}{1-n_{1} n_{2} \cos \alpha-\left(m^{2}-n_{1}^{2} n_{2}^{2} \sin ^{2} \alpha\right)^{\frac{1}{9}}}
$$

$\S 10$. We now proceed to give a second investigation of the value of

$$
\text { - } \mathrm{F}\left(n_{1}, n_{2}, \alpha\right)=\int \frac{d \omega}{\left(1-n_{1} \cos \theta_{1}\right)\left(1-n_{2} \cos \theta_{2}\right)}
$$

where the integration is taken over a sphere of unit radius.

Let the plane containing the radius $\mathrm{OP}$ and the radius $\mathrm{O} u_{1}$ make an angle $\phi$ with the plane containing $\mathrm{O} u_{1}$ and $\mathrm{O} u_{2}$, as shown in fig. 2. Then we have

$\cos \theta_{2}=\cos \theta_{1} \cos \alpha+\sin \theta_{1} \sin \alpha \cos \phi$, while

$$
d \omega=\sin \theta_{1} d \theta_{1} d \phi
$$

Fig. 2.

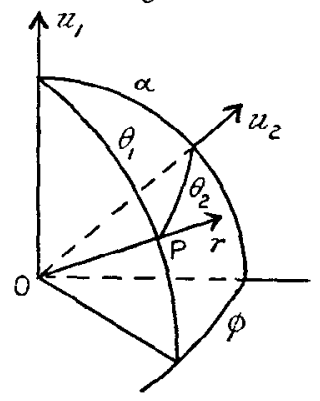


Hence

$$
F=\iint \frac{\sin \theta_{1} d \theta_{1} d \phi}{\left(1-n_{1} \cos \theta_{1}\right)\left(1-n_{2} \cos \alpha \cos \theta_{1}-n_{2} \sin \alpha \sin \theta_{1} \cos \phi\right)}
$$

where $\phi$ goes from 0 to $2 \pi$ and $\theta_{1}$ goes from 0 to $\pi$.

By $\S 7$ we see that

$$
\begin{aligned}
\int_{0}^{2 \pi} \frac{d \phi}{1-n_{2} \cos \alpha \cos \theta_{1}-n_{2} \sin \alpha \sin \theta_{1} \cos \phi} \\
=\frac{2 \pi}{\left\{\left(1-n_{2} \cos \alpha \cos \theta_{1}\right)^{2}-n_{2}^{2} \sin ^{2} \alpha \sin ^{2} \theta_{1}\right\}^{3}}
\end{aligned}
$$

provided that the result is real. But

$$
\begin{aligned}
\left(1-n_{2} \cos \alpha \cos \theta_{1}\right)^{2}- & n_{2}^{2} \sin ^{2} \alpha \sin ^{2} \theta_{1} \\
& =\left(\cos \alpha-n_{2} \cos \theta_{1}\right)^{2}+\sin ^{2} \alpha\left(1-n_{2}^{2}\right),
\end{aligned}
$$

and hence this quantity is always positive, since $n_{2}^{2}<1$. The integration is therefore valid.

Replacing $\sin ^{2} \theta_{1}$ by $1-\cos ^{2} \theta_{1}$ in the $\phi$-integral, we find $\mathrm{F}=\int_{0}^{\pi} \frac{2 \pi \sin \theta_{1} d \theta_{1}}{\left(1-n_{1} \cos \theta_{1}\right)\left(1-n_{2}^{2} \sin ^{2} \alpha-2 n_{2} \cos \alpha \cos \theta_{1}+n_{2}^{2} \cos ^{2} \theta_{1}\right)^{\frac{1}{2}}}$.

To reduce the integral to a simpler form, put

$$
1-n_{1} \cos \theta_{1}=1 / x, \quad \cos \theta_{1}=(x-1) / n_{1} x .
$$

Then

$$
\sin \theta_{1} d \theta_{1}=-d x / n_{1} x^{2}
$$

and $x=\left(1-n_{1}\right)^{-1}$ when $\theta=0$ and $x=\left(1+n_{1}\right)^{-1}$ when $\theta=\pi$. Making these substitutions, we easily find

where

$$
\mathrm{F}=2 \pi \int_{\mathbf{A}}^{\mathrm{B}} \mathrm{X}^{-\frac{2}{2}} d x
$$

$$
\begin{aligned}
& \mathrm{X}=p^{2} x^{2}-2 w n_{2}\left(n_{2}-n_{1} \lambda\right)+n_{2}^{2} \\
& p^{2}=n_{1}^{2}+n_{2}^{2}-2 n_{1} n_{2} \lambda-n_{1}{ }^{2} n_{2}{ }^{2}+n_{1}{ }^{2} n_{2}{ }^{2} \lambda^{2} . . \\
& \lambda=\cos \alpha \text {. . . . . . . . . . } \\
& \mathrm{A}=\left(1+n_{1}\right)^{-1}, \quad \mathrm{~B}=\left(1-n_{1}\right)^{-1} .
\end{aligned}
$$

and

Hence

$$
\mathrm{F}=\frac{2 \pi}{p} \log \mathrm{Y}
$$

where

$$
\log \mathrm{Y}=\left[\log \left\{p^{2} x-n_{2}\left(n_{2}-n_{1} \lambda\right)+p \mathrm{X}^{\frac{1}{2}}\right\}\right]_{\mathbf{A}}^{\mathbf{B}} .
$$


Using (29), we easily find that when $x=\mathrm{B}=\left(1-n_{1}\right)^{-1}$

$$
\mathrm{X}^{\frac{1}{2}}=n_{1}\left(1-n_{2} \lambda\right)\left(1-n_{1}\right)^{-1},
$$

and that when $x=\mathrm{A}=\left(1+n_{1}\right)^{-1}$

Thus

$$
\mathrm{X}^{\frac{1}{2}}=n_{1}\left(1+n_{2} \lambda\right)\left(1+n_{1}\right)^{-1} \text {. }
$$

$$
\mathrm{Y}=\frac{\left(1+n_{1}\right)\left\{p^{2}-n_{2}{ }^{2}+n_{1} n_{2} \lambda+n_{1} n_{2}^{2}-n_{1}^{2} n_{2} \lambda+p n_{1}\left(1-n_{2} \lambda\right)\right\}}{\left(1-n_{1}\right)\left\{p^{2}-n_{2}{ }^{2}+n_{1} n_{2} \lambda-n_{1} n_{2}^{2}+n_{1}{ }^{2} n_{2} \lambda+p n_{1}\left(1+n_{2} \lambda\right)\right\}}
$$

When we substitute for $p^{2}$ from (29), we find that both numerator and denominator contain $n_{1}$ as a factor. When this factor is removed, we have

$$
\mathrm{Y}=\frac{\left(1+n_{1}\right)\left\{n_{1}+n_{2}{ }^{2}-n_{1} n_{2}{ }^{2}-n_{2} \lambda-n_{2} n_{2} \lambda+n_{1} n_{2}{ }^{2} \lambda+p\left(1-n_{2} \lambda\right)\right\}}{\left(1-n_{1}\right)\left\{n_{1}-n_{2}{ }^{2}-n_{1} n_{2}{ }^{2}-n_{2} \lambda+n_{1} n_{2} \lambda+n_{1} n_{2}^{2} \lambda+p\left(1-n_{2} \lambda\right)\right\}} .
$$

On carrying out the multiplications, we find that the five terms on the right side of (29) occur in both numerator and denominator. Replacing these terms by $p^{2}$, we obtain

$$
\begin{aligned}
\mathrm{Y} & =\frac{\left(1-n_{1} n_{2} \lambda\right)\left(n_{1}-n_{2} \lambda\right)+p\left(1-n_{1} n_{2} \lambda+n_{1}-n_{2} \lambda\right)+p^{2}}{\left(1-n_{1} n_{2} \lambda\right)\left(n_{1}-n_{2} \lambda\right)+p\left(1-n_{1} n_{2} \lambda-n_{1}+n_{2} \lambda\right)-p^{2}} \\
& =\frac{\left(1-n_{1} n_{2} \lambda+p\right)\left(n_{1}-n_{2} \lambda+p\right)}{\left(1-n_{1} n_{2} \lambda-p\right)\left(n_{1}-n_{2} \lambda+p\right)} \\
& =\frac{1-n_{1} n_{2} \lambda+p}{1-n_{1} n_{2} \lambda-p}
\end{aligned}
$$

Since, by (29) and (27), $p^{2}=m^{2}-n_{1}^{2} n_{2}^{2} \sin ^{2} \alpha$ and since $. \lambda=\cos \alpha$, we obtain, finally,

$$
\begin{aligned}
& \mathrm{F}\left(n_{1}, n_{2}, \alpha\right)= \\
& \left.\frac{2 \pi}{\left(m^{2}-n_{1}^{2} n_{2}^{2} \sin ^{2} \alpha\right)^{\frac{1}{2}}} \log \frac{1-n_{1} n_{2} \cos \alpha+\left(m^{2}-n_{1}{ }^{2} n_{2}{ }^{2} \sin ^{2} \alpha\right)^{\frac{1}{2}}}{1-n_{1} n_{2} \cos \alpha-\left(m^{2}-n_{1}^{2} n_{2}{ }^{2}\right.} \sin ^{2} \alpha\right)^{\frac{1}{3}}
\end{aligned}
$$

This result agrees with that found in $\S 9$.

$\S 11$. We can now return to the electrical problem and obtain an expression for the energy radiated when the velocity of the sphere is impulsively changed.

Since, by (8)

$$
\mathrm{W}=\mathrm{U}_{0}\left\{(2 \pi)^{-1}\left(1-n_{1} n_{2} \cos \alpha\right) \mathrm{F}\left(n_{1}, n_{2}, \alpha\right)-2\right\}
$$

we have, by (28)

$$
\begin{aligned}
& \mathrm{W} / \mathrm{U}_{0}= \\
& \frac{1-n_{1} n_{2} \cos \alpha}{\left(m^{2}-n_{1}^{2} n_{2}^{2} \sin ^{2} \alpha\right)^{\frac{3}{3}}} \log \frac{1-n_{1} n_{2} \cos \alpha+\left(m^{2}-n_{1}^{2} n_{2}^{2} \sin ^{2} \alpha\right)^{\frac{1}{3}}}{1-n_{1} n_{2} \cos \alpha-\left(m^{2}-n_{1}^{2} n_{2}^{2} \sin ^{2} \alpha\right)^{\frac{1}{2}}}-2 .
\end{aligned}
$$


In terms of the velocities the expression becomes

$$
\begin{gathered}
\mathrm{W} / \mathrm{U}_{0}= \\
\frac{v^{2}-u_{1} u_{2} \cos \alpha}{\left(v^{2} w^{2}-u_{1}^{2} u_{2}^{2} \sin ^{2} \alpha\right)^{\frac{2}{4}}} \log \frac{v^{2}-u_{1} u_{2} \cos \alpha+\left(v^{2} v^{2}-u_{1}^{2} u_{2}^{2} \sin ^{2} \alpha\right)^{\frac{1}{2}}}{v^{2}-u_{1} u_{2} \cos \alpha-\left(v^{2} w^{2}-u_{1}^{2} u_{2}^{2} \sin ^{2} \alpha\right)^{\frac{1}{2}}}-2 .
\end{gathered}
$$

It is easily seen that the values of $W$ found in $\$ 4$ for $\alpha=0$ and for $\alpha=n$ may be deduced from the general expression for $W$.

It is convenient to write $\mathrm{W} / \mathrm{U}_{0}$ in the form

where

$$
\frac{W}{U_{0}}=\frac{1}{Z} \log \frac{1+Z}{1-Z}-2
$$

$$
\mathrm{Z}=\frac{\left(v^{2} w^{2}-u_{1}^{2} u_{2}^{2} \sin ^{2} \alpha\right)^{\frac{1}{2}}}{v^{2}-u_{1} u_{2} \cos \alpha}
$$

The expression for $\mathrm{W} / \mathrm{U}_{0}$ has no meaning when $\mathrm{Z}$, which is positive, is greater than unity. When $\mathrm{Z}$ is less than unity, we have

$$
\frac{\mathrm{W}}{\mathrm{U}_{0}}=2\left\{\frac{\mathrm{Z}^{2}}{3}+\frac{\mathrm{Z}^{4}}{5}+\frac{\mathrm{Z}^{6}}{7}+\ldots\right\} \text {. }
$$

Since all the terms of this series are positive, $W / U_{0}$ increases as $\mathrm{Z}$ increases.

If we write $u_{1}{ }^{2}+u_{2}{ }^{2}-2 u_{1} u_{2} \cos \alpha$ for $w^{2}$, we find

$$
Z^{2}=1-\frac{\left(v^{2}-u_{1}^{2}\right)\left(v^{2}-u_{2}^{2}\right)}{\left(v^{2}-u_{1} u_{2} \cos \alpha\right)^{2}}
$$

Hence $Z$ increases as $\alpha$ increases from 0 to $\pi$. Thus for given values of $u_{1}$ and $u_{2}$, the radiated energy, $W$, increases as the angle between the directions of $u_{1}$ and $u_{2}$ increases, since both $\imath_{1}$ and $u_{2}$ are less than $v$.

For given values of $u_{1}$ and $u_{2}$, the maximum value of $\mathrm{Z}$ occurs when $\alpha=\pi$. Then we have

$$
Z_{\pi}=\frac{v\left(u_{1}+u_{2}\right)}{v^{2}+u_{1} u_{2}}=1-\frac{\left(v-u_{1}\right)\left(v-u_{2}\right)}{v^{2}+u_{1} u_{2}},
$$

and thus $Z_{\pi}$ is positive and less than unity. Collecting these results we see that for all values of $\alpha$ and for all permissible values of $u_{1}$ and $u_{2}, Z$ is positive and less than unity.

$\$ 12$. Since the loss of energy arises from the change in the velocity of the sphere, it will be convenient to express $W$ as directly as possible in terms of $w$, the change of velocity. As we require two other quantities to fix the circumstances of the problem, we take $u$ and $\psi$ where $2 u$ is the resultant of $\mathrm{u}_{1}$ and $\mathrm{u}_{2}$ and $\psi$ is the angle between the positive 
directions of $\mathbf{u}$ and $\mathbf{w}$. For convenience, we write

$$
u=n v . \text {. . . . . . . }
$$

The relations which $u_{1}, u_{2}$, and $\alpha$ bear to $u, w$, and $\psi$ are shown in fig. 3.

From the three equations

$$
\begin{aligned}
& w^{2}=u_{1}^{2}+u_{2}^{2}-2 u_{1} u_{2} \cos \alpha, \\
& u_{1}^{2}=u^{2}+\frac{1}{4} w^{2}-u w \cos \psi, \\
& u_{2}^{2}=u^{2}+\frac{1}{4} w^{2}+u w \cos \psi,
\end{aligned}
$$

Fig. 3.

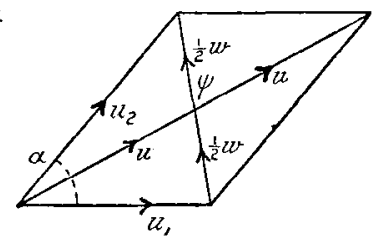

we obtain

$$
\begin{aligned}
& u_{1} u_{2} \cos \alpha=u^{2}-\frac{1}{4} w^{2}, \\
& \text { or } \quad n_{1} n_{2} \cos \alpha=n^{2}-\frac{1}{4} m^{2} .
\end{aligned}
$$

Writing down two equivalent expressions for the area of the parallelogram, we have

$$
u_{1} u_{2} \sin \alpha=u w \sin \psi
$$

or

Hence

$$
n_{1} n_{2} \sin \alpha=n m \sin \psi \text {. }
$$

or

$$
v^{2} w^{2}-u_{1}^{2} u_{2}^{2} \sin ^{2} \alpha=w^{2}\left(v^{2}-u^{2} \sin ^{2} \psi\right),
$$

$$
m^{2}-n_{1}^{2} n_{2}^{2} \sin ^{2} \alpha=m^{2}\left(1-n^{2} \sin ^{2} \psi\right) \text {. }
$$

When these values are inserted in the results of $\S 11$, we obtain

$\frac{\mathrm{W}}{\mathrm{U}_{0}}=\frac{1-n^{2}+\frac{1}{4} m^{2}}{m\left(1-n^{2} \sin ^{2} \psi\right)^{\frac{1}{2}}} \log \frac{1-n^{2}+\frac{1}{4} m^{2}+m\left(1-n^{2} \sin ^{2} \psi\right)^{\frac{3}{3}}}{1-n^{2}+\frac{1}{4} m^{2}-m\left(1-n^{2} \sin ^{2} \psi\right)^{\frac{1}{3}}}-2$,

or

$\frac{\mathrm{W}}{\mathrm{U}_{0}}=\frac{v^{2}-u^{2}+\frac{1}{4} w^{2}}{w\left(v^{2}-u^{2} \sin ^{2} \psi\right)^{\frac{1}{2}}} \log \frac{v^{2}-u^{2}+\frac{1}{4} w^{2}+w\left(v^{2}-u^{2} \sin ^{2} \psi\right)^{\frac{1}{3}}}{v^{2}-u^{2}+\frac{1}{4} v^{2}-w\left(v^{2}-u^{2} \sin ^{2} \psi\right)^{\frac{3}{3}}}-2$.

$\$ 13$. We now pass on to calculate the momentum radiated in the pulse generated by an impulsive change of velocity of the spbere. It will not be necessary to employ the integral calculus, since the momentum can be deduced on dynamical principles from quantities which are already known.

To change the velocity of the sphere impulsively from $\mathbf{u}_{1}$ to $u_{2}$ it is necessary to apply a force $F_{12}$ to the sphere as long as the pulse is passing over the sphere. When the pulse is clear of the sphere the force is no longer required. While the pulse is passing over the sphere, $\boldsymbol{F}_{12}$ probably changes both in direction and in magnitude, but we are not concerned 
with the value of $F_{12}$ at any instant, as we shall eliminate $F_{12}$ from the equations.

I et $\mathrm{U}_{1}, \mathrm{~T}_{1}$, and $\mathrm{U}_{2}, \mathrm{~T}_{2}$ be the electric and magnetic energies of the sphere when moving with the velocities $u_{1}$ and $u_{2}$, and let $W_{1}$ be the energy radiated when the velocity $u_{1}$ is suddenly destroyed, and $W_{2}$ the energy radiated when $u_{2}$ is destroyed. Since the force which stops the sphere does no work, the total energy in the electromagnetic field is unchanged. Before the sphere is stopped the energy is $\mathrm{U}_{1}+\mathrm{T}_{1}$, and after it is stopped the energy is $\mathrm{U}_{0}+\mathrm{W}_{1}$, where $\mathrm{U}_{0}$ is the electrostatic energy. Hence

and

$$
\begin{aligned}
& \mathrm{U}_{1}+\mathrm{T}_{1}=\mathrm{U}_{0}+\mathrm{W}_{1} \\
& \mathrm{U}_{2}+\mathrm{T}_{2}=\mathrm{U}_{0}+\mathrm{W}_{2} .
\end{aligned}
$$

The values of $W_{1}$ and $W_{2}$, which may be deduced from the results of $\S 4$, are given by

$$
\begin{aligned}
\mathrm{W}_{1} & =\mathrm{U}_{0}\left(\frac{v}{u_{1}} \log \frac{v+u_{1}}{v-u_{1}}-2\right) \\
& =\mathrm{U}_{0}\left(\frac{2 n_{1}{ }^{2}}{3}+\frac{2 n_{1}{ }^{4}}{5}+\frac{2 n_{1}{ }^{6}}{7}+\ldots\right) . \\
\mathrm{W}_{2} & =\mathrm{U}_{0}\left(\frac{v}{u_{2}} \log \frac{v+u_{2}}{v-u_{2}}-2\right) . . .
\end{aligned}
$$

Sizce $u_{1}=n_{1} v$, we can write

$$
\mathrm{W}_{1}=\mathrm{U}_{0}\left\{\frac{1}{n_{1}} \log \frac{1+n_{1}}{1-n_{1}}-2\right\} . .
$$

\begin{tabular}{|c|c|c|c|}
\hline$n_{1}$. & $\mathrm{W}_{1} / \mathrm{U}_{0}$ & $n_{1}$ & $\mathbf{W}_{1} / \mathrm{U}_{0^{*}}$ \\
\hline $0 \cdot 1$ & $0 \cdot 00671$ & 0.6 & $0 \cdot 31049$ \\
\hline 0.2 & 0.02733 & $0 \cdot 7$ & 0.47800 \\
\hline 0.3 & 0.06346 & 0.8 & $0 \cdot 74653$ \\
\hline 0.4 & $0 \cdot 11824$ & 0.85 & 0.95565 \\
\hline 0.5 & $0 \cdot 19722$ & 0.9 & $1 \cdot 27160$ \\
\hline
\end{tabular}

If we prefer to do so, we may express $W_{1}$ in terms of $\frac{1}{2} m_{0} v^{2}$, by the formula $\mathrm{U}_{0}=\frac{3}{2} \cdot \frac{1}{2} m_{0} v^{2}$, where $m_{0}$ is the electromagnetic mass for infinitesimal speeds.

$$
\text { TABLE I._-Values of } \frac{W_{1}}{U_{0}} \text {. }
$$


$\S 14$. Now consider the impulsive change of velocity from $\mathfrak{u}_{1}$ to $\mathfrak{u}_{2}$. Before the force $\mathbf{F}_{12}$ acted the energy in the electromagnetic field was $U_{1}+T_{1}$, and after the force has acted the energy is $\mathrm{U}_{2}+\mathrm{T}_{2}+\mathrm{W}$. Hence, by $\S 13$, the gain of energy is $W_{2}-W_{1}+W$, and this must be equal to the work done by the force. Thus, in vector notation,

$$
\begin{aligned}
\mathrm{W}_{2}-\mathrm{W}_{1}+\mathrm{W} & =\int \mathrm{F}_{12} \mathrm{u}_{2} d t \\
& =\mathrm{u}_{2} \int \mathrm{F}_{12} d t, . . . .
\end{aligned}
$$

since $\mathrm{u}_{2}$ is constant, the last expression being the scalar product of $\mathbf{u}_{2}$ and $\int \mathbf{F}_{12} d t$.

Similarly if $\mathbf{F}_{21}$ be the force required to change the velocity from $\mathfrak{u}_{2}$ to $\mathfrak{u}_{1}$, we have

$$
\mathrm{W}_{1}-\mathrm{W}_{2}+\mathrm{W}=\mathfrak{u}_{1} \int \mathbf{F}_{21} d t, . . .
$$

since the energy radiated is the same for the change from $u_{2}$ to $u_{1}$ as for the change from $u_{1}$ to $u_{2}$.

From these equations of energy we pass to the equations of momentum. When the sphere is in steady motion, the resultant momentum of the electromagnetic field is in the same direction. as the velocity of the sphere. If $\mathbf{M}_{1}$ be the momentum when the velocity is $\boldsymbol{u}_{1}$ and $\mathbf{M}_{2}$ when the velocity is $\mathbf{u}_{2}$, it is known that *

$$
\begin{aligned}
. \mathrm{M}_{1} & =\frac{\mathrm{U}_{0}}{2 u_{1}}\left\{\frac{v^{2}+u_{1}^{2}}{v u_{1}} \log \frac{v+u_{1}}{v-u_{1}}-2\right\} . . \\
\mathrm{M}_{2} & =\frac{\mathrm{U}_{0}}{2 u_{2}}\left\{\frac{v^{2}+u_{2}^{2}}{v u_{2}} \log \frac{v+u_{2}}{v-u_{2}}-2\right\} . .
\end{aligned}
$$

Since $u_{1}=n_{1} v$, we can write

$$
\begin{aligned}
\mathrm{M}_{1} & =\frac{\mathrm{U}_{0}}{v}\left[\frac{1}{2}\left(1+\frac{1}{n_{1}^{2}}\right) \log \frac{1+n_{1}}{1-n_{1}}-\frac{1}{n_{1}}\right] \\
& =\frac{\mathrm{U}_{0}}{v}\left\{\frac{4 n_{1}}{1.3}+\frac{8 n_{1}^{3}}{3.5}+\frac{12 n_{1}^{5}}{5.7}+\ldots\right\} .
\end{aligned}
$$

If we prefer to do so, we may express $M_{1}$ in terms of $m_{0} v$ by means of the formula $\mathrm{U}_{0} / v=3 m_{0} v / 4$, where $m_{0}$ is the electromagnetic mass for infinitely slow motion.

* These results may be deduced from $\S 19$ of my previous paper, Phil. Mag. Jan. 1907. 
Motion of an Electrified Sphere.

TABLE II.-Values of $\frac{\mathrm{M}_{1} v}{\mathrm{U}_{0}}$.

\begin{tabular}{|c|c|c|c|}
\hline$n_{2}$ & $\mathrm{M}_{1} v / \mathrm{U}_{\mathrm{D}^{*}}$ & $n_{1}$ & $\mathrm{M}_{2} v / \mathrm{U}_{0^{\circ}}$ \\
\hline 0.1 & 0.1339 & 0.6 & 0.9519 \\
0.2 & 0.2710 & 0.7 & 1.2087 \\
0.3 & 0.4153 & 0.8 & 1.5652 \\
0.4 & 0.5715 & 0.85 & 1.8183 \\
0.5 & 0.7465 & 0.9 & 2.1787 \\
\hline
\end{tabular}

Let $\mathbf{P}$ be the momentum in the pulse, so that

$$
\mathbf{P}=\frac{\mu \mathrm{K}}{4 \pi} \iiint \mathrm{VEH} d x d y d z, . \quad . \quad .
$$

where VEH denotes the vector product of the electric and magnetic forces and the integration extends throughout the volume of the pulse. Then, since the momentum of the electromagnetic field is changed from $\mathbf{M}_{1}$ to $\mathbf{M}_{2}+\mathbf{P}$ by the action of the force $\mathbf{F}_{12}$,

$$
\mathbf{M}_{2}-\mathbf{M}_{1}+\mathbf{P}=\left\{\mathbf{F}_{12} d t . \quad . \quad . .\right.
$$

When the change of velocity of the sphere is effected in the reverse direction, both $\mathbf{E}$ and $\mathbf{H}$ in the pulse are simply reversed in direction without change of magnitude, as appears from (1) and (2), and hence $\mathbf{P}$ remains unchanged. Hence we have

$$
\mathbf{M}_{1}-\mathbf{M}_{2}+\mathbf{P}=\int \mathbf{F}_{21} d t . \quad . \quad .
$$

Combining these equations with (35) and (36), and writing

$$
\mathbf{P}=\mathbf{P}_{1}+\mathbf{P}_{2} \text {, }
$$

where $\mathbf{P}_{\mathbf{1}}$ is in the same direction as $\mathbf{u}_{1}$ and $\mathbf{P}_{2}$ in the same direction as $u_{2}$, we have

$$
\begin{aligned}
& \mathfrak{u}_{2}\left(\mathbf{M}_{2}-\mathbf{M}_{1}+\mathbf{P}_{1}+\mathbf{P}_{2}\right)=\mathrm{W}_{2}-\mathrm{W}_{1}+\mathrm{W} \\
& \mathfrak{u}_{1}\left(\mathbf{M}_{1}-\mathbf{M}_{2}+\mathbf{P}_{1}+\mathbf{P}_{2}\right)=\mathrm{W}_{1}-\mathrm{W}_{2}+\mathrm{W} .
\end{aligned}
$$

Working out the scalar products, we obtain

$$
\begin{aligned}
& P_{1} \cos \alpha+P_{2}=M_{1} \cos \alpha-M_{2}+\left(W_{2}-W_{1}+W\right) u_{2}^{-1}=X_{1} \\
& P_{1}+P_{2} \cos \alpha=M_{2} \cos \alpha-M_{1}+\left(W_{1}-W_{2}+W\right) u_{1}^{-1}=X_{2} .
\end{aligned}
$$


Solving these equations, we have

$$
P_{1}=\frac{X_{2}-X_{1} \cos \alpha}{\sin ^{2} \alpha}, \quad P_{2}=\frac{X_{1}-X_{2} \cos \alpha}{\sin ^{2} \alpha} .
$$

The magnitude of $\mathrm{P}$ is given by

$$
\begin{aligned}
\mathrm{P}^{2} & =\mathrm{P}_{1}{ }^{2}+\mathrm{P}_{2}{ }^{2}+2 \mathrm{P}_{1} \mathrm{P}_{2} \cos \alpha \\
& =\mathrm{P}_{2} \mathrm{X}_{1}+\mathrm{P}_{1} \mathrm{X}_{2} \\
& =\frac{\mathrm{X}_{1}{ }^{2}+\mathrm{X}_{2}{ }^{2}-2 \mathrm{X}_{1} \mathrm{X}_{2} \cos \alpha}{\sin ^{2} \alpha}
\end{aligned}
$$

If |we substitute for $\mathrm{M}_{1}, \mathrm{M}_{2}, \mathrm{~W}_{1}$, and $\mathrm{W}_{2}$ the values given in (37), (38), (32), and (33), and write

$$
\log \frac{v+u_{1}}{v-u_{1}}=\mathrm{L}_{1}, \quad \log \frac{v+u_{2}}{v-u_{2}}=\mathrm{L}_{2},
$$

and make a slight re-arrangement of the factor of $L_{1}$, we find that

$$
\begin{aligned}
\mathrm{P}_{2}+\mathrm{P}_{1} \cos \alpha=\frac{\mathrm{W}}{u_{2}}+\frac{\mathrm{U}_{0}}{v} & {\left[\left\{\frac{\left(v^{2}-u_{1}^{2}\right) \cos \alpha}{2 u_{1}^{2}}-\frac{v^{2}-u_{1} u_{2} \cos \alpha}{u_{1} u_{2}}\right\} \mathrm{L}_{1}\right.} \\
+ & \left.\frac{v^{2}-u_{2}^{2}}{2 u_{2}^{2}} \mathrm{I}_{2}+\frac{v\left(u_{1}-u_{2} \cos \alpha\right)}{u_{1} u_{2}}\right] .
\end{aligned}
$$

Similarly, we find

$$
\begin{aligned}
\mathrm{P}_{1}+\mathrm{P}_{2} \cos \alpha=\frac{\mathrm{W}}{u_{1}}+ & \frac{\mathrm{U}_{0}}{v}\left[\left\{\frac{\left(v^{2}-u_{2}^{2}\right) \cos \alpha}{2 u_{2}^{2}}-\frac{v^{2}-u_{1} u_{2} \cos \alpha}{u_{1} u_{2}}\right\} \mathrm{L}_{2}\right. \\
& \left.+\frac{v^{2}-u_{1}^{2}}{2 u_{1}^{2}} \mathrm{~L}_{1}+\frac{v\left(u_{2}-u_{1} \cos \alpha\right)}{u_{1} u_{2}}\right] . \quad(43 a)
\end{aligned}
$$

We can, without difficulty, write out the value of $\mathrm{P}^{2}$, but the expression becomes very complicated in the general case. We shall therefore consider its value in special cases only.

The component parallel to $\mathrm{v}_{1}$ of the momentum in the pulse is $\mathrm{P}_{1}+\mathrm{P}_{2} \cos \alpha$. If we make $u_{2}=0$, we obtain the momentum in the pulse which is formed when $u_{1}$, the initial velocity of the sphere, is suddenly destroyed. The expression for $\mathrm{P}_{1}+\mathrm{P}_{2} \cos \alpha$ (not $\left.\mathrm{P}_{2}+\mathrm{P}_{1} \cos \alpha\right)$ becomes indeterminate when we put $\imath_{\mathfrak{g}}=0$, but this difficulty can be overcome by first expanding $\mathrm{L}_{2}$ in the form $2 n_{2}+2 n_{2} 3 / 3+\ldots$, and then putting $n_{2}=0$ in the terms which do not cancel. Using the value of $W_{1}$ given by (34) and using $u_{1}=n_{1} v$, we find for 
the resultaut momentum in the pulse formed on stopping the sphere

$$
\begin{aligned}
\mathrm{P}_{\mathrm{l}} & =\frac{\mathrm{U}_{0}}{v}\left\{\left(\frac{3}{2 n_{1}^{2}}-\frac{1}{2}\right) \log \frac{1+n_{1}}{1-n_{1}}-\frac{3}{n_{1}}\right\} . . \\
& =\frac{\mathrm{U}_{0}}{v}\left\{\frac{4 n_{1}^{3}}{3.5}+\frac{8 n_{1}^{5}}{5.7}+\frac{12 n_{1}{ }^{7}}{7.9}+\ldots\right\} .
\end{aligned}
$$

This result agrees with that given in $\$ 19$ of my earlier paper. In Table III. the numerical values of $\mathrm{P}_{1} v / \mathrm{U}_{0}$ are given as well as the ratio of $\mathrm{P}_{1}$ to $\mathrm{M}_{1}$, i.e. the ratio of the momentum carried off in the pulse to the momentum of the field before the velocity is destroyed. It will be easily seen from (39) and (44) that, when $n_{1}$ is very small, $P_{1} / M_{1}=n_{1}{ }^{2} 5$. Un the other hand, when $n_{1}$ approaches unity, only the logarithmic parts of the expressions for $P_{1}$ and $M_{1}$ need be considered, and we find that although both $P_{1}$ and $M_{1}$ tend to infinity, their ratio $P_{1} / M_{1}$ tends to unity.

\begin{tabular}{|c|c|c|c|c|c|}
\hline$n_{1}$ & $\mathbf{P}_{1^{10} / \mathbf{U}_{0}}$ & $\mathrm{P}_{1} / \mathrm{M}_{1}$ & $n_{1}$ & $\mathbf{P}_{1} v / \mathbf{U}_{0}$ & $\mathrm{P}_{1} / \mathrm{M}_{1}+$ \\
\hline 0 & 0 & 0 & $0 \cdot 6$ & 0.08308 & 0.08728 \\
\hline 0.1 & 0.00027 & 0.00201 & 0.7 & $0 \cdot 15699$ & $0 \cdot 12988$ \\
\hline $0 \cdot 2$ & $0.002: 21$ & 0.00816 & 0.8 & $0 \cdot 30113$ & $0 \cdot 19239$ \\
\hline 0.3 & 0.00780 & 0.01878 & 0.85 & $0 \cdot 43029$ & 0.23664 \\
\hline $0 \cdot 4$ & 0.01977 & 0.03459 & $0 \cdot 9$ & 0664711 & 0.29702 \\
\hline 0.5 & 0.04237 & $0 \cdot 05676$ & $1 \cdot 0$ & $\infty$ & 1.00000 \\
\hline
\end{tabular}

TABLE III.-Values of $\frac{\mathrm{P}_{1} v}{\mathrm{U}_{0}}$ and of $\frac{\mathrm{P}_{1}}{\mathrm{M}_{1}}$.

If the "impulse" of the force which stops the motion of the sphere be $I_{1}$, then

$$
M_{1}-I_{1}=P_{1},
$$

and hence, by (39) and (44),

$$
\begin{aligned}
\mathbf{I}_{1} & =\mathrm{M}_{1}-\mathrm{P}_{1} \\
& =\frac{\mathrm{U}_{0}}{v}\left\{\left(1-\frac{1}{n_{1}^{2}}\right) \log \frac{1+n_{1}}{1-n_{1}}+\frac{2}{n_{1}}\right\} .
\end{aligned}
$$

Since $\left(1-n_{1}\right) \log \left(1-n_{1}\right)$ tends to zero as $n_{1}$ tends to unity, it follows that the limiting value of $\mathrm{I}$ is $2 \mathrm{U}_{0} / v$.

Thus it appears that if a charged sphere moving at the 
speed of light be arrested by an obstacle, it only communicates a finite part of its infinite momentum to the obstacle. From Table II. we see that the momentum communicated to the obstacle isequal to that possessed by the sphere when moving at a speed of about $0.87 v$.

The "impulse" of the force required to suddenly give the sphere a velocity equal to that of light is $P_{1}+M_{1}$, and thus is infinite.

When the sphere is stopped, the pulse ceases to cut the sphere after a time $2 a / v$, and then no force is required to hold the sphere at rest.

But when the sphere is started into motion at the speed of light, the sphere is always just enclosed in its own pulse, and consequently the force required to maintain its motion does not vanish until the motion has continued for an infinite time.

The component in the direction opposite to the sphere's motion of the electric force in the pulse is *

$$
\mathrm{E}=\frac{\mathrm{Q} u \sin \theta}{2 \mathrm{~K} r a(v-u \cos \theta)} \sin \theta
$$

provided $r$ be very great compared with $a$. When $u=v$ and $\theta$ is very small,

$$
\mathrm{E}=\frac{\mathrm{Q}}{\mathrm{K} r a} .
$$

If $x$ be measured along the direction of motion from the centre of the sphere, the retarding mechanical force experienced by the sphere in consequence of the action of the pulse tends to the value

$$
\int_{-a}^{+a} \frac{\mathrm{Q}}{\mathrm{K} r a} \cdot \frac{\mathrm{Q} d x}{2 a}=\frac{\mathrm{Q}^{2}}{\mathrm{~K} a r}
$$

or to the value

$$
\frac{\mathrm{Q}^{2}}{\mathrm{~K} a r t},
$$

when $t$, the time since the sphere was started, tends to infinity. I stated this result in a review of Dr. Heaviside's 'Electromagnetic Theory' in the 'Physical Review,' July 1900 , where, however, by some error the resuit is printed $\mathrm{Q}^{2} / 2$ avt $\dagger$.

When a charged sphere is suddenly set into motion with velocity $u$ at the time $t=0$, the force required to maintain that velocity does not cease until $t=2 a /(v-u), i$. e, until the

* Phil. Mag. January 1907, p. 124.

+ The printers did not furnish me with a "proof" of the review. 
pulse generated by the sudden change of velocity no longer cuts the sphere. Dr. Paul Hertz * has obtained a complete solution for the force required in starting the sphere at any time from $t=0$ to $t=2 a /(v-u)$, and, by extending his method, I have found the force required to stop the sphere $\dagger$.

$\S 15$. The momentum in the pulse can also be obtained by direct integration. Since in the pulse $\mathrm{H}=v \mathrm{KVRE}$, it follows that VEH $=v K$ VEVRE, where $\mathbf{R}$ is a unit-vector along the radius. But $\mathbf{E}$ is perpendicular to $\mathbf{R}$, and thus $\mathrm{VEH}=v \mathrm{KE}^{2} \mathbf{R}$. Hence VEH is in the same direction as $\mathbf{R}$. Thts, by (40) the momentum in the pulse is

$$
\mathbf{P}=\frac{\mathrm{K}}{4 \pi v} \iiint \mathrm{E}^{2} \mathbf{R} d x d y d z,
$$

the integration extending throughout the volume of the pulse.

Since the thickness of the pulse is $2 a$, it follows that, if $r$ ke the radius of the pulse and $d \omega$ an element of solid angle,

$$
\mathrm{P}=\frac{\mathrm{K}}{2} \frac{r^{2} a}{2 \pi v} \int \mathrm{E}^{2} \mathrm{R} d \omega
$$

The value of $\mathrm{E}^{2}$ is given in (3). For brevity we write

$$
\begin{gathered}
v-u_{1} \cos \theta_{1}=h_{1}, \quad v-u_{2} \cos \theta_{2}=h_{2}, \\
v^{2}-u_{1} u_{2} \cos \alpha=\mathrm{S} .
\end{gathered}
$$

Noting that $\mathrm{Q}^{2} / 2 \mathrm{~K} a=\mathrm{U}_{0}$, we obtain

$$
\mathbf{P}=\frac{\mathrm{U}_{0}}{4 \pi v} \int\left(\frac{2 \mathrm{~S}}{h_{1} h_{2}}-\frac{v^{2}-u_{1}^{2}}{h_{1}{ }^{2}}-\frac{v^{2}-u_{2}{ }^{2}}{h_{2}{ }^{2}}\right) \mathbf{R} d \omega .
$$

The component of $\mathbf{P}$ parallel to $\mathfrak{u}_{2}$ is $P_{2}+P_{1} \cos \alpha$, with the notation of $\S 14$, and thus, since the angle between $\mathbf{R}$ and $\mathrm{u}_{2}$ is $\theta_{2}$, we have

$$
\mathrm{P}_{2}+\mathrm{P}_{1} \cos \alpha=\frac{\mathrm{U}_{0}}{4 \pi v} \int\left(\frac{2 \mathrm{~S}}{h_{1} h_{2}}-\frac{v^{2}-u_{1}^{2}}{h_{1}^{2}}-\frac{v^{2}-u_{2}^{2}}{h_{2}^{2}}\right) \cos \theta_{2} d \omega .
$$

* Untersuchungen ïber unstetige Bewegungen eines Electrons.

† " On the Force required to stop a Moving Electrified Sphere," Proc. Royal Society, A. vol. Ixxix. p. 550. In this paper 1 have given a slietch of Dr. Hertz's method and have stated his results. 
Now $\cos \theta_{2}=\left(\eta-h_{2}\right) / u_{2}$, and hence

$$
\int \frac{\cos \theta_{2} d \omega}{h_{1} h_{2}}=\frac{1}{u_{2}}\left\{v \int \frac{d x}{h_{1} h_{2}}-\int \frac{d \omega}{h_{1}}\right\} .
$$

But, by (7),

$$
v \int \frac{d \omega}{h_{1} h_{2}}=\frac{1}{v} \mathrm{~F}\left(n_{1}, n_{2}, \alpha\right)
$$

and

$$
\int \frac{d \omega}{h_{1}}=2 \pi \int_{0}^{\pi} \frac{\sin \theta_{1} d \theta_{1}}{v-u_{1} \cos \theta_{1}}=\frac{2 \pi}{u_{1}} \mathrm{~L}_{1},
$$

where

$$
\mathrm{L}_{1}=\log \frac{v+u_{1}}{v-u_{1}} \text { and } \mathrm{L}_{2}=\log \frac{v+u_{2}}{v-u_{2}} \text {. }
$$

With the notation of $\S 10$,

and thus

$$
\cos \theta_{2}=\cos \theta_{1} \cos \alpha+\sin \theta_{1} \sin x \cos \phi,
$$

$$
\int \frac{\cos \theta_{2} d \omega}{h_{1}{ }^{2}}=\iint \frac{\left(\cos \theta_{1} \cos \alpha+\sin \theta_{1} \sin \alpha \cos \phi\right) \sin \theta_{1} d \theta_{1} d \phi}{h_{1}{ }^{2}},
$$

where $\phi$ goes from 0 to $2 \pi$ and $\theta_{1}$ from 0 to $\pi$. The term involving $\cos \phi$ vanishes on integration with respect to $\phi$. Writing $\left(v-h_{1}\right) / u_{1}$ for $\cos \theta_{1}$, we obtain

$$
\begin{aligned}
\int \frac{\cos \theta_{2} d \omega}{h_{1}{ }^{2}} & =\frac{2 \pi \cos \alpha}{u_{1}}\left\{v \int_{0}^{\pi} \frac{\sin \theta_{1} d \theta_{1}}{h_{1}^{2}}-\int_{0}^{\pi} \frac{\sin \theta_{1} d \theta_{1}}{h_{1}}\right\} \\
& =\frac{2 \pi \cos \alpha}{u_{1}}\left\{\frac{2 v}{v^{2}-u_{1}{ }^{2}}-\frac{\mathrm{L}_{1}}{u_{1}}\right\} .
\end{aligned}
$$

Writing $\left(v-h_{2}\right) / u_{2}$ for $\cos \theta_{2}$, we obtain

$$
\begin{aligned}
\int \frac{\cos \theta_{2} d \omega}{h_{2}{ }^{2}} & =\frac{2 \pi}{u_{2}}\left\{v \int_{0}^{\pi} \frac{\sin \theta_{2} d \theta_{2}}{h_{2}{ }^{2}}-\int_{0}^{\pi} \frac{\sin \theta_{2} d \theta_{2}}{h_{2}}\right\} \\
& =\frac{2 \pi}{u_{2}}\left\{\frac{2 v}{v^{2}-u_{2}{ }^{2}}-\frac{L_{4}}{u_{2}}\right\} .
\end{aligned}
$$

Collecting these results, and substitating for $\mathrm{F}\left(n_{1}, n_{\mathfrak{g}}, \alpha\right)$ the value derived from (8), we have

$$
\begin{aligned}
\mathbf{P}_{2}+\mathrm{P}_{1} \cos \alpha=\frac{\mathrm{W}}{u_{2}}+\frac{\mathrm{U}_{0}}{v}[\{ & \left.\frac{\left(v^{2}-u_{1}^{2}\right) \cos \alpha}{2 u_{1}^{2}}-\frac{v^{2}-u_{1} u_{2} \cos \alpha}{u_{1} u_{2}}\right\} \mathrm{L}_{1} \\
& \left.+\frac{v^{2}-u_{2}^{2}}{2 u_{2}^{2}} \mathrm{~L}_{2}+\frac{v\left(u_{1}-u_{2} \cos \alpha\right)}{u_{1} u_{2}}\right] .
\end{aligned}
$$


This result is identical with (43), which was obtained in $\S 14$ by dynamical principles.

$\S 16$. When $w$, the change of velocity, is small compared with $\left(v^{2}-u^{2}\right)^{\frac{1}{2}}$, where $2 u$ is the resultant of $\mathbf{u}_{1}$ and $\mathbf{u}_{2}$, we can obtain approximate expressions for $W$ and for $P$ which may be usefúl for many purposes.

The energy radiated when $v$ is small is easily deduced from the expression given in $\$ 12$. Since, when $y / x$ is less than 1 ,

$$
\underset{\sim}{x} \log \frac{x+y}{x-y}-2=2\left(\frac{y^{2}}{3 x^{2}}+\frac{y^{4}}{5 x^{4}}+\ldots\right)
$$

it follows that, when $m$ or $w / v$ is small,

$$
\mathrm{W}=\mathrm{U}_{0} \frac{2 m^{2}\left(1-n^{2} \sin ^{2} \psi\right)}{3\left(1-n^{2}+\frac{1}{4} m^{2}\right)^{2}} .
$$

But $\mathrm{U}_{0}=\mathrm{Q}^{2} / 2 \mathrm{~K} a=\mu v^{2} \mathrm{Q}^{2} / 2 a$, and thus as far as terms in $w^{2}$,

$$
\mathrm{W}=\frac{2 \mathrm{U}_{0} w^{2}\left(1-n^{2} \sin ^{2} \psi\right)}{3 v^{2}\left(1-n^{2}\right)^{2}}=\frac{\mu Q^{2} w^{2}\left(1-n^{2} \sin ^{2} \psi\right)}{3 a\left(1-n^{2}\right)},
$$

a value identical with that obtained when $w$ is treated as small throughout *. Thus the effect of a given change of velocity depends upon the initial velocity. If $W_{0}$ be the energy radiated when the velocity is changed from 0 to $v$, we have $\dagger$, as far as $w^{2}$,

$$
\mathrm{W}_{\mathrm{e}}=\frac{\boldsymbol{\mu} \mathrm{Q}^{2} w^{2}}{3 a}
$$

and hence

$$
\frac{W}{W_{0}}=\frac{1-n^{2} \sin ^{2} \psi}{\left(1-n^{2}\right)^{2}}
$$

The following table shows the value of $\mathrm{W} / \mathrm{W}_{0}$ for a few values of $n$ and of $\psi$.

It will be seen from the table that the anglerbetween the initial velocity and the change of velocity has only a small effect when the initial velocity is small. For initial velocities less than $\frac{1}{5} v$ the energy radiated does not differ by as much as 10 per cent. from the energy radiated when the initial

* Phil. Mag. Jan. 1907, p. 146.

† Phil. Mag. Jan. 1907, p. $13 i$ (or $\$ 13$ above).

Phil. Mag. S. 6. Vol. 17. No. 97. Jan. 1909. 
velocity is zero, the small change of velocity being the same in either case.

TABLe IV.-Values of $\frac{W}{W_{0}}$.

\begin{tabular}{|c|c|c|c|c|}
\hline$n$. & $\begin{array}{l}\psi=0, \\
\psi=180^{\circ} .\end{array}$ & $\begin{array}{l}\psi=30^{\circ} . \\
\psi=150^{\circ} .\end{array}$ & $\begin{array}{l}\psi=60^{\circ} \\
\psi=120^{\circ}\end{array}$ & $\psi=90^{\circ}$ \\
\hline $0 \cdot 1$ & 1.020 & 1.018 & $1 \cdot 013$ & $1 \cdot 010$ \\
\hline 0.2 & $1 \cdot 085$ & $1 \cdot 074$ & 1.053 & $1 \cdot 049$ \\
\hline 0.3 & $1 \cdot 208$ & $1 \cdot 180$ & $1 \cdot 126$ & 1.099 \\
\hline $0 \cdot 4$ & 1.417 & $1 \cdot 361$ & $1 \cdot 247$ & $1 \cdot 190$ \\
\hline 0.5 & 1.778 & $1 \cdot 667$ & $1 \cdot 444$ & $1 \cdot 333$ \\
\hline 0.6 & $2 \cdot 441$ & $2 \cdot 222$ & $1 \cdot 782$ & 1.562 \\
\hline 07 & 3.845 & $3 \cdot 374$ & $2 \cdot 432$ & 1.961 \\
\hline $0 \cdot 8$ & $7 \cdot 716$ & $6 \cdot 481$ & $4 \cdot 012$ & 2.778 \\
\hline $0 \cdot 85$ & 12986 & $10 \cdot 640$ & 5.949 & $3 \cdot 604$ \\
\hline 0.9 & $27 \cdot 701$ & $22 \cdot 091$ & 10.873 & $5 \cdot 263$ \\
\hline
\end{tabular}

$\S 17$. In $\S 32$ of my previous paper I obtained approximate expressions for the components of the radiated momentum, in directions parallel and perpendicular to $\mathfrak{u}$, by treating $\mathbf{w}$ as small from the beginning. It will be convenient to express these results in terms of $m_{0}=2 \mu \mathrm{Q}^{2} / 3 a$, the electromagnetic mass of the sphere for infinitesimal speeds.

If $\mathrm{P}_{u}{ }^{*}$ be the component in the direction of $\mathrm{u}$, then

$$
\begin{aligned}
\mathrm{P}_{u}= & \frac{m_{0} w^{2}}{v} \cdot \frac{3}{8 n^{4}}\left[\log \frac{1+n}{1-n}-\frac{2 n\left(3-5 n^{2}\right)}{3\left(1-n^{2}\right)^{2}}\right. \\
& \left.\quad-\sin ^{2} \psi\left\{\frac{3+n^{2}}{2} \log \frac{1+n}{1-n}-\frac{n\left(9-12 n^{2}-n^{4}\right)}{3\left(1-n^{2}\right)^{2}}\right\}\right] \\
= & \frac{m_{0} v^{2}}{v}\left(\mathrm{~A}-\mathrm{B} \sin ^{2} \psi\right),
\end{aligned}
$$

where $A$ and $B$ are functions of $n$.

For numerical calculations it is convenient to express $P_{u}$ in the form of a series, which we may use when $n$ is small. Thus

$$
\begin{aligned}
& \mathbf{P}_{u}=\frac{m_{0} w^{2}}{v}\left[\left\{\frac{1.2}{5} n+\frac{2.3}{7} n^{3}+\frac{3.4}{9} n^{5}+\ldots\right\}\right. \\
& \left.-\frac{2}{2} \sin ^{2} \Psi\left\{\frac{3}{3.5} n+\frac{34}{5.7} n^{3}+\frac{117}{7.9} n^{5}+\frac{276}{9.11} n^{7}+\ldots\right\}\right] .
\end{aligned}
$$

* In the previous paper (Phil. Mag. Jan. 1907) the momentum in the direction of $\mathbf{u}$ was denoted by $P_{1}$ and the momentum perpendicular to $\mathbf{u}$ by $\mathrm{P}_{2}$. 
If $\mathrm{P}_{t}$ be the transverse component of the momentum, $i . e$. the component perpendicular to $u$ in the direction making an acute angle with $\mathrm{w}$,

$$
\begin{aligned}
P_{t} & =\frac{m_{0} w^{2}}{16 v} \cdot \frac{3 \sin 2 \psi}{16 n^{4}}\left[\frac{2 n\left(3-2 n^{2}\right)}{3\left(1-n^{2}\right)}-\log \frac{1+n}{1-n}\right] \\
& =\frac{m_{0} w^{2}}{v} \mathrm{C} \sin 2 \psi,
\end{aligned}
$$

where $\mathrm{C}$ is a function of $n$.

Expressing the result in a series, we have

$$
\mathrm{P}_{t}=\frac{m_{0} w^{2}}{v} \cdot \frac{\sin 2 \psi}{4}\left[\frac{n}{5}+\frac{2 n^{3}}{7}+\frac{3 n^{5}}{9}+\frac{4 n^{7}}{11}+\ldots\right] .
$$

I have verified that the values of $P_{1}$ and $P_{2}$ given in $\S 14$ lead to the above formula for $P_{t}$ by expanding all the quantities in (43) in powers of $w$ as far as $w^{2}$, the process involving a good deal of labour. The verification of the formula for $\mathrm{P}_{t}$ requires the expansions to be carried as far as $w^{3}$, and this led to expressions which were so complicated that I abandoned the work.

Tables V. and VI., which have been prepared for me by my friend and former colleague R. S. Cole, show the values

\begin{tabular}{|c|c|c|c|c|c|c|c|}
\hline \multirow[b]{2}{*}{$n$. } & \multirow[b]{2}{*}{ A. } & \multirow[b]{2}{*}{ B. } & \multicolumn{5}{|c|}{$v \mathrm{P}_{u} / m_{0} w^{2}$} \\
\hline & & & $\psi=0^{\circ}$. & $\begin{array}{l}\psi=30^{\circ} \\
\psi=150^{\circ}\end{array}$ & $\begin{array}{l}\psi=45^{\circ} \\
\psi=135^{\circ}\end{array}$ & $\begin{array}{l}\psi=60^{\circ} . \\
\psi=120 .\end{array}$ & $\psi=90^{\circ}$. \\
\hline 0.1 & 0.0409 & 0.0105 & 00409 & 0.0382 & 0.0356 & 00330 & 0.0304 \\
\hline 02 & 0.0873 & 0.0242 & 00873 & 0.0813 & 0.0752 & 0.0692 & 0.0631 \\
\hline 0.3 & 0.1468 & 0.0457 & $0 \cdot 1468$ & $0 \cdot 1354$ & $0 \cdot 1240$ & $0 \cdot 1125$ & 0.1011 \\
\hline 04 & 0.2322 & 0.0835 & 0.2322 & $0 \cdot 2114$ & 0.1905 & $0 \cdot 1696$ & $0 \cdot 1487$ \\
\hline 0.5 & 03695 & $0 \cdot 1559$ & 0.3695 & 0.3305 & 0.2915 & 0.2525 & 0.2135 \\
\hline 06 & 0.6204 & 0.3099 & 0.6204 & 0.5430 & 0.4655 & 0.3880 & 0.3105 \\
\hline 0.7 & $1 \cdot 1680$ & 0.6924 & $1 \cdot 1680$ & $0 \cdot 9948$ & $0 \cdot 8217$ & 0.6486 & 0.4755 \\
\hline 08 & $2 \cdot 7651$ & 1.9461 & 27651 & $2 \cdot 2786$ & $1 \cdot 7921$ & $1 \cdot 3056$ & 0.8190 \\
\hline 0.85 & $5 \cdot 0427$ & $3 \cdot 8667$ & $5 \cdot 0427$ & $4 \cdot 0760$ & $3 \cdot 1094$ & $2 \cdot 1427$ & $1 \cdot 1760$ \\
\hline $0 \cdot 9$ & 11.6575 & $9 \cdot 7421$ & $11 \cdot 6575$ & $9 \cdot 2220$ & 67865 & $4 \cdot 3510$ & 1.9154 \\
\hline
\end{tabular}
of $v \mathrm{P}_{u} / m_{0} w^{2}$ and of $v \mathrm{P}_{t} / m_{0} w^{2}$ for a few values of $n$ and of $\psi$.

TABLE V. Values of $\frac{v \mathrm{P}_{z}}{m_{0} w^{3}}=\mathrm{A}-\mathrm{B} \sin ^{2} \psi_{\text {. }}$ 
TABLE VI.-Values of $\frac{v \mathrm{P}_{t}}{m_{0} w^{2}}=\mathrm{C} \sin 2 \psi$.

\begin{tabular}{|c|c|c|c|c|c|}
\hline \multirow[b]{2}{*}{$n$} & \multirow{2}{*}{ c. } & \multicolumn{4}{|c|}{$v \mathrm{P}_{t} / m_{0} w^{2}$} \\
\hline & & $\begin{array}{l}\psi=0^{\circ} \\
\psi=90^{\circ} .\end{array}$ & $\begin{array}{l}\psi=15^{\circ} . \\
\psi=75^{\circ} .\end{array}$ & $\begin{array}{l}\psi=30^{\circ} . \\
\psi=60^{\circ} .\end{array}$ & $\psi=45^{\circ}$ \\
\hline 0.1 & 0.0051 & 0 & 0.0025 & 0.0044 & 0.0051 \\
\hline $0 \cdot 2$ & $0-0106$ & 0 & 0.0053 & 0.0092 & 0.0106 \\
\hline $0 \cdot 3$ & 0.0172 & 0 & 0.0086 & $0 \cdot 0149$ & 0.0172 \\
\hline 0.4 & $0-0256$ & 0 & 0.0128 & 0.0222 & 0.0256 \\
\hline $0 \cdot 5$ & 00375 & 0 & 0.0187 & 0.0325 & 0.0375 \\
\hline 0.6 & $0-0560$ & 0 & $0 \cdot 0280$ & $0 \cdot 0485$ & $0 \cdot 0560$ \\
\hline $0 \cdot \bar{T}$ & 0.0888 & 0 & 0.0444 & 0.0769 & $0 \cdot 0888$ \\
\hline 0.8 & $0-1606$ & 0 & 00803 & 0.1391 & $0 \cdot 1606$ \\
\hline 0.85 & 0.2382 & 0 & $0 \cdot 1191$ & $0-2063$ & 0.2382 \\
\hline $0 \cdot 9$ & 0.4039 & 0 & 0.2020 & $0 \cdot 3498$ & 0.4039 \\
\hline
\end{tabular}

$\S 18$. I shall now consider the energy and the momentum which are radiated when the impulsive change of velocity affects the direction but not the magnitude of the velocity. If the direction of the velocity is changed by the angle $\alpha$, we have

$$
\begin{aligned}
& u_{2}=u_{1}=n_{1} v, \\
& w^{2}=2 u_{1}^{2}(1-\cos \alpha)=4 n_{1}^{2} v^{2} \sin ^{2} \frac{1}{2} \alpha, \\
& \left(v^{2} w^{2}-u_{1}^{2} u_{2}^{2} \sin ^{2} \alpha\right)^{\frac{1}{2}}=2 n_{1} v^{2} \sin \frac{1}{2} \alpha\left(1-n_{1}^{2} \cos ^{2} \frac{1}{2} \alpha\right)^{\frac{1}{4}} .
\end{aligned}
$$

Hence, by $\S 11$, we have

where

$$
\frac{\mathrm{W}}{\tilde{\mathrm{U}}_{0}}=\frac{1}{\mathrm{Z}} \log \frac{1+\mathrm{Z}}{\mathrm{I}-\mathrm{Z}}-2,
$$

$$
Z=\frac{2 n_{1} \sin \frac{1}{2} \alpha\left(1-n_{1}^{2} \cos ^{2} \frac{1}{2} \alpha\right)^{\frac{1}{2}}}{1-n_{1}^{2} \cos \alpha}
$$

When $n_{1}$ is small, the numerical calculation of $\mathrm{W} / \mathrm{U}_{0}^{*}$ is best made by the series

$$
\frac{\mathrm{W}}{\mathrm{U}_{0}}=2\left\{\frac{\mathrm{Z}^{2}}{3}+\frac{\mathrm{Z}^{4}}{5}+\frac{\mathrm{Z}^{6}}{7}+\ldots\right\}
$$

which, by $\S 11$, is always valid when $n_{1}$ is less than unity.

If the denominator of each term of the last series be expanded in powers of $n_{1}{ }^{2}$, we obtain a double series in $n_{1}{ }^{2}$. This can be rearranged in the form of a single power series in $n_{1}{ }^{2}$, 
provided that the double series remains convergent when the modulus of every term is substituted for the term itself. This will be the case if

$$
\frac{4 n_{1}^{2} \sin ^{2} \frac{1}{2} \alpha\left(1+n_{1}^{2} \cos ^{2} \frac{1}{2} \alpha\right)}{\left(1-n_{1}^{2}(\cos \alpha \mid)^{2}\right.}<1
$$

where $|\cos \alpha|$ is the modulus of $\cos \alpha$.

The quantity on the left increases with $n_{1}$, and hence the inequality will be satisfied if $n_{1}$ be less than the least real value obtained from the equation

$$
4 n_{1}^{2} \sin ^{2} \frac{1}{2} \alpha\left(1+n_{1}^{2} \cos ^{2} \frac{1}{2} \alpha\right)=\left(1-n_{1}^{2} ! \cos \alpha \mid\right)^{2} .
$$

When $\alpha$ is not greater than $\frac{1}{2} \pi$ this equation leads to $(\sqrt{2}-1)^{\frac{3}{2}}$, or $0 \cdot 643 \ldots$ as the value of $n$. When $\alpha$ is greater than $\frac{1}{2} \pi$, the value is $\sqrt{\overline{2}}-1$, or $0.414 \ldots$ Hence, if $n<\sqrt{2}-1$ the double series can certainly be rearranged as a single series for all values of $\alpha$.

On rearranging this series, we obtain

$$
\begin{aligned}
\frac{W}{\mathrm{U}_{0}}= & 8 n_{1} \mathrm{~S}^{2}\left\{\frac{1}{3}+\left(5-3 \mathrm{~S}^{2}\right) \frac{n_{1}^{2}}{3.5}+\left(35-42 \mathrm{~S}^{2}+16 \mathrm{~S}^{4}\right) \frac{n_{1}{ }^{4}}{3.5 .7}\right. \\
& \left.+\left(105-189 \mathrm{~S}^{2}+144 \mathrm{~S}^{4}-40 \mathrm{~S}^{6}\right) \frac{n_{1}{ }^{6}}{5.7 .9}+\ldots\right\},
\end{aligned}
$$

where $S=\sin \frac{1}{2} \alpha$.

\begin{tabular}{|c|c|c|c|c|c|c|c|}
\hline$n_{1}$. & $\alpha=0^{\circ}$. & $a=30^{\circ}$. & $\alpha=60^{\circ}$. & $\alpha=90^{\circ}$. & $\alpha=120^{\circ}$ & $a=150^{\circ}$ & $\alpha=180^{\circ}$ \\
\hline $0 \cdot 1$ & 0 & 0.0018 & 0.0067 & 0.0134 & $0 \cdot 0201$ & 0.0250 & 0.0268 \\
\hline 0.2 & 0 & 0.0074 & 0.0276 & 0.0547 & 0.0818 & $0 \cdot 1013$ & $0 \cdot 1084$ \\
\hline 0.3 & 0 & 0.0177 & 0.0650 & $0 \cdot 1281$ & 0.1895 & 0.2333 & $0 \cdot 2492$ \\
\hline 0.4 & 0 & 0.0338 & 0.1235 & $0 \cdot 2404$ & 03315 & $0 \cdot 4293$ & 0.4572 \\
\hline 0.5 & 0 & 0.0588 & 0.2118 & $0 \cdot 4050$ & 0.5325 & 07038 & $0 \cdot 7465$ \\
\hline $0 \cdot 6$ & 0 & 0.0983 & 0.3464 & $0 \cdot 6454$ & 0.9082 & 1.0820 & $1 \cdot 1423$ \\
\hline 0.7 & 0 & $0 \cdot 1653$ & 0.5623 & 1.0087 & 1.3779 & $1 \cdot 6125$ & 1.6922 \\
\hline 0.8 & 0 & 0.2968 & 0.9475 & 1.6030 & $2 \cdot 1021$ & $2 \cdot 4040$ & $2 \cdot 5043$ \\
\hline 0.85 & 0 & 0.4221 & $1 \cdot 2773$ & $2 \cdot 0704$ & 26433 & 29864 & 3.0911 \\
\hline 0.9 & 0 & 0.6540 & $1 \cdot 8161$ & $2 \cdot 7765$ & $3 \cdot 4 \cdot 285$ & 3.8008 & 3.9216 \\
\hline
\end{tabular}

Mr. R. S. Cole has prepared Table VII., giving the values of $\mathrm{W} / \mathrm{U}_{0}$ for some values of $n_{1}$ and of $\alpha$.

TABLE VII.-Values of $\underset{\mathrm{U}_{0}}{\mathrm{~W}}$. 
The radiated momentum is, by symmetry, along the direction bisecting the angle between the initial and final velocities. In this case the two components $P_{1}$ and $P_{2}$, which are parallel to $u_{1}$ and $u_{2}$, have equal values, and thus

$$
\mathrm{P}=2 \mathrm{P}_{1} \cos \frac{1}{2} \alpha .
$$

Hence, since $\mathrm{M}_{2}=\mathrm{M}_{1}$ and $\mathrm{W}_{2}=\mathrm{W}_{1}$, we find by $\S 14$ that

$$
\begin{aligned}
P & =2 \cos \frac{1}{2} \alpha \frac{M_{1}(\cos \alpha-1)+W / u_{1}}{1+\cos \alpha} \\
& =\left(\frac{W}{u_{1}}-2 M_{1} \sin ^{2} \frac{1}{2} \alpha\right) \sec \frac{1}{2} \alpha .
\end{aligned}
$$

Hence

$$
\frac{\mathrm{P} v}{\overline{\mathrm{U}}_{0}}=\left[\frac{\mathrm{W}}{n_{1} \overline{\mathrm{U}}_{0}^{-}}-\frac{2 \mathrm{M}_{1} v}{\overline{\mathrm{U}}_{0}} \sin ^{2} \frac{1}{2} \alpha\right] \sec \frac{1}{2} \alpha . \quad .
$$

If we wish, we can express. $P$ in terms of $m_{0} v$ by means of the formula $\mathrm{U}_{0} / v=3 m_{0} v / 4$, where $m_{0}$ is the electromagnetic mass for infinitesimal speeds.

If we make use of the series (39) for $\mathrm{M}_{1}$ and combine it with the series (45) for $\mathrm{W} / \mathrm{U}_{0}$, we obtain the series

$$
\begin{aligned}
\stackrel{\mathrm{P} v}{\mathrm{U}_{0}}=8 n_{1}{ }^{2} \mathrm{~S}^{2} \sqrt{1-\mathrm{S}^{2}}\left\{\frac{1}{5}+\left(26-16 \mathrm{~S}^{2}\right) \frac{n_{1}{ }^{2}}{3.5 .7}\right. \\
\left.+\left(85-104 \mathrm{~S}^{2}+40 \mathrm{~S}^{4}\right) \frac{n_{1}{ }^{4}}{5.7 .9}+\ldots\right\},
\end{aligned}
$$

where, as before, $S=\sin \frac{1}{2} \alpha$. This series is certainly valid when $n_{1}<0.414 \ldots$.

In Table VIII., which was prepared by Mr. A. J. Bamford of Emmanuel College, the values of $\mathrm{P} v / \mathrm{U}_{0}$ for $n_{1}=0 \cdot 1, n=0 \cdot 2$, and $n_{1}=0.3$ were calculated by aid of this series. For the remaining values of $n_{1}, \mathrm{P} v / \mathrm{U}_{0}$ was calculated by (46) from the values of $\mathrm{W} / \mathrm{U}_{0}$ given in Table VII. and those of $\mathrm{M}_{1} v / \mathrm{U}_{0}$. given in Table II.

When $\alpha=\pi$, so that the motion is just reversed, the value of $P$ given by (46) becomes indeterminate since then $\cos \frac{1}{2} \alpha=0$ and $W=2 u_{1} M_{1}$, as appears from (13) and (39). But in this case symmetry demands that $\mathbf{P}$ should vanish. 
TABLE VIII.-Values of $\frac{\mathrm{P} v}{\mathrm{U}_{0}}$.

\begin{tabular}{|c|c|c|c|c|c|c|c|}
\hline$n_{1}$. & $\alpha=0^{\circ}$. & $\alpha=30^{\circ}$ & $\alpha=60^{\circ}$ & $\alpha=90^{\circ}$. & $\alpha=120^{\circ}$ & $\alpha=150^{\circ}$. & $\alpha=180^{\circ}$. \\
\hline 0.1 & 0 & 0.00010 & 0.00035 & 0.00057 & 0.00060 & 0.00039 & 0 \\
0.2 & 0 & 0.00087 & 0.00289 & 0.00468 & 0.00493 & 0.00316 & 0 \\
0.3 & 0 & 0.00312 & 0.01031 & 0.01654 & 0.01723 & 0.01095 & 0 \\
0.4 & 0 & 0.0082 & 0.0266 & 0.0417 & 0.0430 & 0.0264 & 0 \\
0.5 & 0 & 0.0182 & 0.0581 & 0.0898 & 0.0905 & 0.0564 & 0 \\
0.6 & 0 & 0.0376 & 0.1171 & 0.1751 & 0.1716 & 0.1046 & 0 \\
0.7 & 0 & 0.0768 & 0.2297 & 0.3285 & 0.3108 & 0.1859 & 0 \\
0.8 & 0 & 0.1670 & 0.4640 & 0.6202 & 0.5596 & 0.3257 & 0 \\
0.85 & 0 & 0.2619 & 0.6854 & 0.8733 & 0.7646 & 0.4381 & 0 \\
0.9 & 0 & 0.4501 & 1.0722 & 1.2817 & 1.0828 & 0.6091 & 0 \\
\hline
\end{tabular}

In conclusion I desire to thank those friends who have assisted me in the preparation of this paper. But for the help of Mr. G. T. Bennett I should have given up the problem as unmanageable. To Mr. R. S. Cole and to my colleague Mr. T. G. Bedford, and to Mr. A. J. Bamford I am indebted for much help in the heavy arithmetical work involved in the construction and verification of the numerical tables. $\mathrm{Mr}$. Bamford has also verified the mathematical work in the paper.

IV. A Modified Theory of Gravitation.

$$
\text { By C. V. Burton, D.Sc.* }
$$

1. SO many questions arise in connexion with any theory $N$ which aims at assigning a dynamical basis for gravitation, that it may conduce to clearness if some elements of this paper are first presented without mathematical treatment.

The theory now put forward is pulsatory, and may in fact be regarded as a development of that which has been suggested by Prof. Hicks $\uparrow$. The æther, even where modified

* Communicated by the Physical Society : read May 8, 1908.

$\dagger$ W. M. Hicks, "On the Problem of two Pulsating Spheres in a Fluid," Proc. Camb. Phil. Soc. iii. p. 277 (Oct. 1879). 